\title{
Turbulent Heat Transfer in Drag-Reducing Channel Flow of Viscoelastic Fluid
}

\author{
Takahiro Tsukahara and Yasuo Kawaguchi \\ Tokyo University of Science \\ Japan
}

\section{Introduction}

The fascinating effects of minute quantities of high-molecular-weight polymer or surfactant, dissolved in water or another Newtonian fluid, on turbulent behaviors have stimulated considerable research. The most striking phenomenon is the reduction of turbulent frictional drag in wall-bounded flows. As has been well known for 60 years since its first demonstration by Toms (1949), drag reduction (DR) up to $80 \%$ can be achieved in appropriate conditions. The application of this phenomenon, also called the "Toms effect", to heat-transport systems such as district heating and cooling systems has recently attracted attention as an energy-saving technology. The pumping power can be largely conserved by adding a few hundred ppm of surfactant solution to water so that the flow becomes laminar-like. One of the most successful applications of DR has been in the Trans-Alaska pipeline, where the desired discharge of an additional million barrels of crude oil per day was accomplished by the use of polymeric additives rather than by constructing additional pumping systems.

On the other hand, heat transfer reduction (HTR) for a drag-reducing flow is also significant and becomes large with increased DR. If conditions that give rise to large DR and small HTR can be identified, then they should be ideal for the transport of heat in thermal systems. Moreover, it is practically important to formulate phenomenological models and semiempirical correlations, similar to those employed in the description of turbulent Newtonian flows, for viscoelastic flows accompanied by DR and HTR.

\subsection{Related studies}

The turbulence modulation of drag-reducing flows is more complicated than that of a Newtonian-fluid flow such as water. Fluid containing drag-reducing additives can be generally treated as a non-Newtonian viscoelastic fluid, as assumed in the present study. A number of fundamental studies on DR have been carried out, in part because the subject lies at the intersection of two complex and important fields of rheology and turbulence. Although it is practically difficult to analyze the interaction between additives and turbulent motions at the molecular level, many key aspects of the drag-reducing flow have gradually been elucidated. For instance, we already know that additives inhibit the transfer of energy from the streamwise to the wall-normal velocity fluctuations, and that the strong vorticity fluctuation near the wall disappears in the drag-reducing flow. There are several reviews (Dimant \& Poreh, 1976; Gyr \& Bewersdorff, 1995; Hoyt, 1990; Lumley, 
1969; Nadolink \& Haigh, 1995; Procaccia et al. , 2008; Shenoy, 1984; White \& Mungal, 2008) that highlight the progress made in understanding this subject.

Recent direct numerical simulations (DNSs) based on various types of constitutive equations have revealed some important characteristics common to turbulent pipe and channel flows subject to DR. Several features observed in experiments have been successfully reproduced by these numerical analyses (Den Toonder et al., 1997; Dimitropoulos et al., 2001; 2005; Jovanović et al., 2006; Li et al., 2006; Sureshkumar \& Beris, 1995; Sureshkumar et al., 1997; Tamano et al., 2007). The authors' group (Yu \& Kawaguchi, 2004; Yu et al., 2004; Yu \& Kawaguchi, 2005; 2006) has simulated viscoelastic fluids by DNS with the Giesekus model. Although there are certain other commonly used models (e.g., FENE-P, Oldroyd-B), we selected the Giesekus model for our study because it can well describe the measured apparent shear viscosity and extensional viscosity of the surfactant solution (cf. Wei et al., 2006). Through these studies, the occurrence of a high level of DR was found to require high elastic energy in a wide buffer layer with large relaxation time, that is, a high Weissenberg number $\left(W e_{\tau}\right)$. More recent numerical studies by Roy et al. (2006) have suggested that, for a drag-reducing polymer solution, the self-sustaining process of wall turbulence becomes weaker owing to the effect of elasticity on the coherent structures. Their analysis also showed that, at small $W e_{\tau}$, elasticity enhances the quasi-streamwise vortex structures. Similarly, Kim et al. (2008) reported that the autogeneration of new hairpin vortices typical of wall turbulence, which are closely related to the buffer layer, can be suppressed by the polymer stresses, thereby resulting in DR.

Although research on drag-reducing flow with heat transfer is important for various kinds of heat-transport systems and interesting from a scientific perspective, there have been very few studies on this issue (Aguilar et al., 1999; 2001; Dimant \& Poreh, 1976; Gasljevic et al., 2007; Li et al., 2004a;b; 2005), particularly in terms of numerical simulations (Gupta et al., 2005; Kagawa, 2008; Yu \& Kawaguchi, 2005). Early experiments presented some empirical models for heat transfer in drag-reducing flows (Dimant \& Poreh, 1976) and showed that the heat-transfer coefficient was reduced at a rate faster than the accompanying DR (Cho \& Hartnett, 1982), and that an analogous reduction of HTR was observed in the case of drag-reducing surfactant solution (Qi et al., 2001). Several attempts to enhance the efficiency of the heat transfer in drag-reducing flows have been reported in the literature (Aly et al., 2006; Li et al., 2001; Qi et al., 2001). For instance, Qi et al. (2003) examined the methodology of temporal heat-transfer enhancement of drag-reducing surfactants in heat exchangers and regaining DR downstream. Nevertheless, the trade-off between the DR and HTR in industrial applications has not been completely understood. Furthermore, the mechanism of the HTR itself in drag-reducing flows has not been clearly established. As mentioned above, predictions of intermediate values of friction and heat transfer are not yet possible, even if the rheological and thermal properties of a relevant fluid are known.

\subsection{Purpose}

In the research presented in this article, we addressed a wide range of issues related to drag-reducing flows by means of DNS on turbulent heat transfer; and we also review previous DNS results (Kagawa, 2008; Tsukahara et al., 2011a; Tsukahara \& Kawaguchi, 2011b) with additional computations. We have considered dilute surfactant solutions, in which the shear-thinning behavior is assumed to be negligible, but elongational viscoelastic effect is taken into account using a method for the extra elastic stresses. To achieve a clearer picture of the role of viscoelasticity, we have used DNS based on the Giesekus viscoelastic-fluid model. 


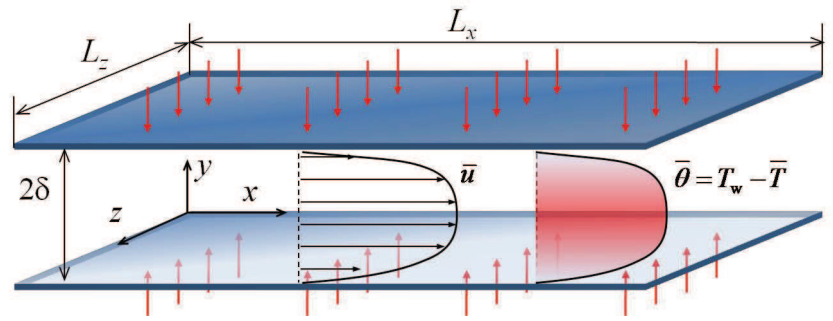

Fig. 1. Configuration of channel flow and heat transfer under thermal boundary condition of uniform heat-flux heating wall, viz. constant streamwise uniform wall temperature gradient.

All fluid properties are considered constant and the heat is treated as passive scalar. The buoyancy effect and the temperature dependence are neglected.

The objectives of this study are (i) to examine the relationship of the thermal field to the drag-reducing (viscoelastic) flows, and (ii) to obtain a semiempirical correlation formula of the heat-transfer reduction rate. For the first objective, the classical layer theory of wall-bounded turbulence is reassessed for drag-reducing flows and also for mean temperature profiles, using the present DNS results. For the second objective, we parametrically investigate the heat-transfer characteristics for rheologically different fluids and for various Prandtl numbers. For the purpose of practical application, simplified forms of equations and phenomenological models are proposed and examined.

\section{Problem description and numerical procedure}

The present subject of interest is the turbulent heat transfer in drag-reducing turbulent flow of (viscoelastic) surfactant solution in a two-dimensional planar channel. The thermal boundary condition considered in this study is constant heat-flux heating on the upper and lower walls of the channel. A detailed description of the subject, together with the relevant properties and governing equations of test fluid, can be found in the literature (Tsukahara et al., 2011a; Tsukahara \& Kawaguchi, 2011b; Yu \& Kawaguchi, 2004; 2005).

For completeness, a brief description of the numerical procedure follows here. For the channel flow, we choose an orthogonal coordinate system $(x, y, z), x$ being the streamwise direction, $y$ the wall-normal direction, and $z$ the spanwise direction. Figure 1 illustrates the flow configuration. In terms of the channel half width, $\delta$, the computational domain involves a rectangular box of dimensions $L_{x} \times 2 \delta \times L z$ with periodic conditions imposed along the horizontal, $x$ and $z$, directions. The periodic boundary condition was adopted in the horizontal $(x$ and $z$ ) directions, and the non-slip boundary condition was imposed on the walls. The turbulent flow that we considered in this study was assumed to be fully developed and driven by the externally imposed pressure gradient in the streamwise direction, but the bulk mean flow rate is dependent on the fluid properties, that is, the magnitude of DR.

\subsection{Rheological properties of viscoelastic fluid}

Since the character of surfactant solution is more complicated than that of polymer solution, no rigorous constitutive equation is available for surfactant solution. However, the rheological properties of the surfactant solution are similar to those of the polymer solution, for example, shear-thinning and extensional thickening, and thus the viscoelastic models for the polymer solution are expected to be applicable to the surfactant case. Therefore, in common with the 
dilute polymer solution, the fluid motion considered here is described by the conventional continuity and momentum equations:

$$
\begin{gathered}
\nabla \cdot \mathbf{u}=0 \\
\rho \frac{\mathrm{D} \mathbf{u}}{\mathrm{D} t}=-\nabla p+\nabla \cdot \boldsymbol{\tau}_{\text {total }}
\end{gathered}
$$

where $\rho$ is the density (assumed constant), $t$ the time, $\mathbf{u}=\{u, v, w\}$ the velocity, $p$ the pressure, and $\tau_{\text {total }}$ the total extra stress tensor. For a homogeneous solution of a Newtonian solvent and a surfactant solute, the extra stress is decomposed as $\tau_{\text {total }}=\tau_{\text {solvent }}+\tau$, with a Newtonian solvent component $\tau_{\text {solvent }}=\rho \eta_{\mathrm{s}} \Delta \mathbf{u}$, where the solvent kinematic viscosity $\eta_{\mathrm{s}}$ is constant, and there is an extra stress-tensor component due to additives, $\tau$. To account for elasticity effects,we employed a viscoelastic Giesekus constitutive equation to model the interaction between the elastic network structures and solvent, following a proposal by Giesekus (1982):

$$
\boldsymbol{\tau}+\lambda\left(\frac{\mathrm{D} \boldsymbol{\tau}}{\mathrm{D} t}-\boldsymbol{\tau} \cdot \nabla \mathbf{u}-\nabla \mathbf{u}^{\mathrm{T}} \cdot \boldsymbol{\tau}\right)+\frac{\alpha \lambda}{\rho \eta_{\mathrm{a}}}(\boldsymbol{\tau} \cdot \boldsymbol{\tau})=\rho \eta_{\mathrm{a}}\left(\nabla \mathbf{u}+\nabla \mathbf{u}^{\mathrm{T}}\right)
$$

where $\lambda, \alpha$, and $\eta_{\mathrm{a}}$ are, respectively, a constant relaxation time, the mobility factor, and the additive contribution to the zero-shear-rate solution kinematic viscosity, $\eta_{0}$. This model is known to describe the power-law regions for viscosity and normal-stress coefficients and it also provides a reasonable description of the elongational viscosity (Bird, 1995). When $\lambda=0$, we obtain the momentum equation for Newtonian fluid with the kinematic viscosity of $\eta_{\mathrm{s}}+$ $\eta_{\mathrm{a}}$. Equation (3) with $\alpha=0$ corresponds to the Oldroyd-B model. Note that, in general, both $\lambda$ and $\eta_{\mathrm{a}}$ can be considered as functions of the stress or other state variables (e.g., temperature), but they will be assumed to be constants in the present study for simplicity.

Kawaguchi et al. (2003) reported that the measured shear viscosities were well demonstrated by the Giesekus model for different temperatures and concentrations of surfactant solution and that, using the model parameters obtained from the correlations, most of the measured shear viscosities agreed with the prediction by the Giesekus model. Yu et al. (2004) adopted this model using parameters that were determined by well-fitting apparent shear viscosities based on measurement using rheometers. Their comparison between numerical and experimental results revealed the rheological properties of a viscoelastic fluid of $75 \mathrm{ppm}$ CTAC (cetyltrimethyl ammonium chloride) solution at $30^{\circ} \mathrm{C}$, namely, $\lambda=0.3$ seconds, $\alpha=0.005$, and the viscosity ratio $\beta=\eta_{\mathrm{s}} / \eta_{0}=0.2$. It should be noted that the contribution of the additive to the solution viscosity was relatively large compared with those obtained or assumed in previous studies on DR. Since many experiments described in the literature were carried out at low concentrations of polymer and achieved significant DRs, most DNSs in the literature employed artificially low values without detailed measurement.

\subsection{Governing equations for velocity field}

To describe the system in a non-dimensional form, let us introduce the dimensionless conformation tensor, $c_{i j}$, with which the instantaneous additive extra stress $\left(\tau_{i j}\right)$ is given as an explicit function:

$$
\tau_{i j}^{+}=\frac{c_{i j}^{+}-\delta_{i j}}{\lambda u_{\tau} / \delta} .
$$


In the equation above, $\delta_{i j}$ is the Kronecker delta. As for a characteristic velocity, the friction velocity $\left(u_{\tau}\right)$ based on the mean pressure gradient in the streamwise direction is chosen:

$$
u_{\tau}=\sqrt{\frac{\tau_{\mathrm{w}}}{\rho}}=\sqrt{-\frac{\delta}{\rho} \frac{\partial \bar{p}}{\partial x}} .
$$

The dimensionless governing equations for an incompressible viscoelastic-fluid flow are the continuity, momentum conservation, and constitutive equations, which can be rewritten in tensor form based on Equations (1)-(3) as follows:

$$
\begin{gathered}
\frac{\partial u_{i}}{\partial x_{i}}=0, \\
\frac{\partial u_{i}^{+}}{\partial t^{\star}}+u_{j}^{+} \frac{\partial u_{i}^{+}}{\partial x_{j}^{\star}}=-\frac{\partial p^{+}}{\partial x_{i}^{\star}}+\frac{\beta}{R e_{\tau}} \frac{\partial}{\partial x_{j}^{\star}}\left(\frac{\partial u_{i}^{+}}{\partial x_{j}^{\star}}\right)+\frac{(1-\beta)}{W e_{\tau}} \frac{\partial c_{i j}^{+}}{\partial x_{j}^{\star}}+\frac{\partial \bar{p}^{+}}{\partial x_{1}^{\star}} \delta_{1 i} . \\
\frac{\partial c_{i j}^{+}}{\partial t^{\star}}+\frac{\partial u_{k}^{+} c_{i j}^{+}}{\partial x_{k}^{\star}}-c_{i k}^{+} \frac{\partial u_{j}^{+}}{\partial x_{k}^{\star}}-\frac{\partial u_{i}^{+}}{\partial x_{k}^{\star}} c_{k j}^{+}+\frac{R e_{\tau}}{W e_{\tau}}\left[c_{i j}^{+}-\delta_{i j}+\alpha\left(c_{i k}^{+}-\delta_{i k}\right)\left(c_{k j}^{+}-\delta_{k j}\right)\right]=0,
\end{gathered}
$$

where $i=1,2$, and 3 indicate the streamwise, wall-normal, and spanwise directions, respectively. The scales used to obtain the non-dimensional variables are $u_{\tau}$ and $\eta_{0}$. The last term on the right-hand side of Equation (7) corresponds to the mean pressure gradient driving the flow. The superscript " $\star$ " is used to denote non-dimensional distances (e.g., $y^{\star}=y / \delta$ ) and the superscript "+" denotes the wall unit.

As suggested by Equation (7), the flow is defined by three control parameters:

$$
\begin{gathered}
\text { Friction Reynolds number: } R e_{\tau}=\frac{u_{\tau} \delta}{\eta_{0}}, \\
\text { Weissenberg number: } W e_{\tau}=\frac{\lambda}{\eta_{0} / u_{\tau}^{2}}, \\
\text { Viscosity ratio: } \beta=\frac{\eta_{\mathrm{s}}}{\eta_{0}} .
\end{gathered}
$$

Table 1 shows a summary of tested parameters for the Newtonian fluid (case 1) and the four different viscoelastic fluids.

\subsection{Governing equation for thermal field}

With the calculated flow field, the instantaneous temperature of $T(x, y, z)$ was obtained by integrating the equation of energy conservation:

$$
\frac{\partial T^{+}}{\partial t^{\star}}+u_{j}^{+} \frac{\partial T^{+}}{\partial x_{j}^{\star}}=\frac{1}{R e_{\tau} \operatorname{Pr}} \frac{\partial^{2} T^{+}}{\partial x_{j}^{\star 2}},
$$

with non-dimensionalization by the friction temperature. In the present simulation, the temperature difference, $\theta\left(=T_{\mathrm{W}}-T\right)$, is introduced with the following operation. As illustrated in Fig. 1, both walls are uniformly heated with constant wall heat flux (but the instantaneous heat flux is time-dependent), so that the statistically averaged temperature 
increases linearly with respect to the $x$ direction. Therefore, $T(x, y, z)$ can be divided into two parts:

$$
T(x, y, z)=\frac{\mathrm{d} T_{\mathrm{m}}}{\mathrm{d} x} x-\theta(x, y, z),
$$

where $T_{\mathrm{m}}$ is the so-called bulk mean temperature defined as follows:

$$
T_{\mathrm{m}}=\frac{\int_{0}^{\delta} \bar{u}\langle T\rangle \mathrm{d} y}{\int_{0}^{\delta} \bar{u} \mathrm{~d} y} .
$$

Here, $\langle T\rangle$ is the temperature averaged in time and in the $z$ direction, while an overbar denotes a quantity also averaged in the $x$ direction. The denominator of Equation (14) corresponds to the bulk mean velocity, $u_{\mathrm{m}}=\int_{0}^{1} \bar{u} \mathrm{~d} y^{\star}$. From the heat flux balance and the present configuration, the streamwise temperature gradient in Equation (13) becomes

$$
\frac{\mathrm{d} T_{\mathrm{m}}^{+}}{\mathrm{d} x^{\star}}=\frac{1}{u_{\mathrm{m}}^{+}}
$$

Thus, with the above transformation, we can arrange the equation for $\theta(x, y, z)$ from Equation (12) into the following form:

$$
\frac{\partial \theta^{+}}{\partial t^{\star}}+u_{j}^{+} \frac{\partial \theta^{+}}{\partial x_{j}^{\star}}=\frac{1}{R e_{\tau} \operatorname{Pr}} \frac{\partial^{2} \theta^{+}}{\partial x_{j}^{\star 2}}+\frac{u}{u_{\mathrm{m}}} .
$$

The boundary conditions for the momentum and thermal fields are:

$$
u_{i}=\theta=0 \text {, at } y=0 \text { and } 2 \delta \text {. }
$$

\subsection{Simulation methodology and parameters}

The current DNS is carried out by employing a finite-difference-method code solving Equations (6)-(8) and (16), but the pressure Poisson equation was solved in Fourier space The implicit dependence of velocity and pressure is decoupled by a fractional-step method. Time integration was performed via the second-order Adams-Bashforth method, but the implicit second-order Crank-Nicolson method was for the viscous terms in the wall-normal direction. A numerical scheme with fourth-order central difference was employed in the $x$ and $z$ directions, and that with second-order accuracy was applied in $y$. The above numerical scheme has been extensively used to study Newtonian channel flow and turbulent heat transfer problems (Kawamura et al., 1998; Kozuka et al., 2009).

It is well known that the addition of a constitutive equation considerably modifies the mathematical character of the resulting system of governing equations, where accumulation of numerical errors during time integration can cause a loss of positive definiteness and a breakdown of the numerical calculations, which can trigger the Hadamard instability (Joseph, 1990). This instability issue in viscoelastic-flow calculations has been well documented in the literature of steady-state viscoelastic flow simulations, and could be overcome by improving the numerical methods (e.g., Basombrío et al., 1991; Fortin \& Fortin, 1989; Sureshkumar \& Beris, 1995). Nevertheless, in view of the high non-linearity of the governing equations, no complete mathematical theory has so far been available on the existence and uniqueness of viscoelastic flows. In addition, the high Weissenberg-number problem 


\begin{tabular}{ccccccccc}
\hline Cases & Fluid & $R e_{\tau}$ & $W e_{\tau}$ & $\beta$ & $P r$ & $R e_{\mathrm{m}}$ & $\eta_{\mathrm{eff}} / \eta_{0}$ & $D R \%$ \\
\hline 1 & Newtonian 150 & 0 & 1.0 & $0.1,1.0,2.0$ & 4650 & 1.000 & - \\
2 & viscoelastic 150 & 10 & 0.5 & $0.1,1.0,2.0$ & 5130 & 0.872 & $20.7 \%$ \\
3a & viscoelastic 150 & 30 & 0.5 & $0.1,1.0,2.0$ & 7900 & 0.690 & $62.8 \%$ \\
$3 \mathrm{~b}$ & viscoelastic 150 & 30 & 0.3 & $0.1,1.0,2.0$ & 8860 & 0.518 & $69.5 \%$ \\
4 & viscoelastic 150 & 40 & 0.5 & $0.1,1.0,2.0$ & 9210 & 0.654 & $71.5 \%$ \\
\hline
\end{tabular}

Table 1. Given and resultant flow parameters

remains an open question (see the review papers of Bird, 1995; Keunings, 1990). The upwind differencing scheme has been found necessary in order to ensure stable calculations. In our simulation, the first/second-order MINMOD scheme was adapted to the convective term in Equation (8). This scheme is a composite flux-limiter scheme (almost identical to the SOUCUP scheme) consisting of the second-order central differencing and first-order upwind schemes (see Zhu \& Rodi, 1991). More details on this can be found by referring to (Yu \& Kawaguchi, 2004).

We considered the turbulent channel flow at a fixed friction Reynolds number of $R e_{\tau}=150$ and investigated the heat-transfer character in the drag-reducing turbulence. The fluid rheological parameters of the Weissenberg number and the viscosity ratio are varied between $W e_{\tau}=0$ and 40 and $\beta=1.0$ and 0.5. Recently, the present authors's group (Kagawa, 2008; Tsukahara \& Kawaguchi, 2011b) performed the DNSs with a Prandtl number of $\mathrm{Pr}=2.0$. In this work, their DNS is extended to include scalar transport with Prandtl numbers of 0.1 and 1.0. A total of 15 cases have been simulated. They are described in Table 1 . In both case 3a and case $3 b$, the same Weissenberg number is given to examine the effect of variance in $\beta$, while the condition change among cases $2,3 \mathrm{a}$, and 4 is variation in $W e_{\tau}$ at a constant $\beta$. The mobility factor of $\alpha=0.001$ is fixed under all DNSs for viscoelastic fluid.

The horizontal computational domain sizes are $L_{x}=12.8 \delta$ and $L_{z}=6.4 \delta$ with discretizing into $128 \times 128 \times 128$ grid points in $x, y$, and $z$, respectively. The dimensionless grid resolutions in the $x$ and $z$ directions are evenly distributed $(\Delta x=0.1, \Delta z=0.05)$. In the wall-normal direction, the non-uniform resolutions are stretched away from the walls, in which $\Delta y$ ranges from 0.0015 at the walls to 0.030 at the channel center. These sizes in wall units, $\Delta x^{+}, \Delta y^{+}$, and $\Delta z^{+}$, correspond to $15.0,0.226-4.52$, and 7.5 , respectively. The time incremental interval, $\Delta t^{+}$, is less than $1.5 \times 10^{-3}$.

\subsection{Representative mean-flow variables}

In Table 1, important resultant characteristics regarding the mean flow are presented prior to the discussion. To achieve reasonable scaling of various turbulent statistics, an effective wall kinematic viscosity $\eta_{\text {eff }}$ was calculated from the proportionality between the total wall shear stress $\tau_{w}$ and the mean velocity gradient at the wall as follows:

$$
\frac{\tau_{w}}{\rho}=\eta_{\text {eff }}\left(\frac{\mathrm{d} \bar{u}}{\mathrm{~d} y}\right)_{y=0} .
$$

The ratio of $\eta_{\text {eff }} / \eta_{0}$ in a viscoelastic flow is generally less than unity because extra stress due to additives occurs in addition to the usual viscous stress. In a Newtonian flow, $\eta_{\text {eff }}=\eta_{0}$. As shown in Table $1, \eta_{\text {eff }} / \eta_{0}$ decreases to near 0.5 in case $3 b$, which is much smaller than in the 


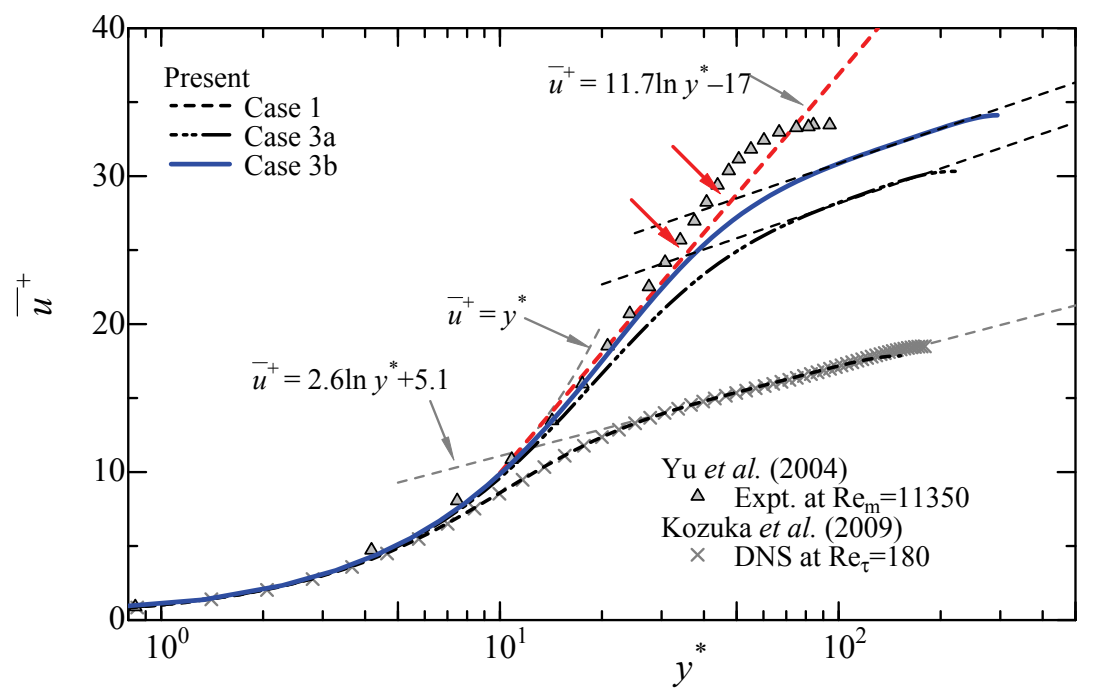

Fig. 2. Mean velocity versus wall-normal position in inner units. Viscous scalings are defined using the effective viscosity. A red arrow indicates the thickness (upper edge) of elastic layer, $y_{\mathrm{e}}$, which is defined by a crossover point of Equation (21) for the elastic layer and Equation (22) for the turbulent core.

other cases. In case 3b, a high level of DR was obtained and found to be comparable to that in case 4 . The percentage of DR rate is defined as follows:

$$
D R \%=\frac{C_{f_{\text {Newt }}}-C_{f_{\text {visc }}}}{C_{f_{\text {Newt }}}} \times 100 \%,
$$

where $C_{f_{\text {visc }}}$ is an obtained friction coefficient and $C_{f_{\text {Newt }}}$ is estimated by the empirical correlation function,

$$
C_{f_{\text {Newt }}}=0.073 \operatorname{Re}_{\mathrm{m}}^{-0.25},
$$

for Newtonian turbulent channel flow (Dean, 1978) at the same value of bulk Reynolds number $R e_{\mathrm{m}}$ (also shown in Table 1). Better quantifications of $D R \%$ (and $H T R \%$ ) were previously proposed in the literature (Gasljevic \& Matthys, 1999; Housiadas \& Beris, 2004), but will not be used here for simplicity.

\section{Results and discussion}

\subsection{Mean velocity profile}

Firstly, we shall briefly discuss the velocity field accompanied by the wall-turbulence modulation and the drag reduction due to the fluid viscoelasticity, prior to any consideration of the temperature field.

Figure 2 presents the mean velocity profile, obtained for the Newtonian flow (case 1) and two different viscoelastic flows (cases $3 a$ and $3 b$ ), in a semi-logarithmic coordinate. Note again that statistics denoted by an overbar are spatially (in $x$ and $z$ ) and temporally averaged. The abscissa is the wall-normal distance normalized as $y^{*}=y u_{\tau} / \eta_{\text {eff }}$. Note that $y^{*}$ for Newtonian flow is equivalent to $y^{+}$. By applying a normalization based on the effective viscosity, velocity 
profiles in the drag-reducing turbulent channel flow are scaled well, as given in the figure. Profiles other than the shown cases have already been described (Tsukahara et al., 2011a; Tsukahara \& Kawaguchi, 2011b) and are not presented here. Also shown is the experimental result obtained by Yu et al. (2004), where $D R \%$ of $51 \%$ was achieved using surfactant solution. The DNS result for Newtonian flow at $R e_{\tau}=150$ by Kozuka et al. (2009) is also included in the figure. The present results are in qualitative agreement with their data.

As is well known, the velocity profiles in drag-reducing flow exhibit a characteristic shape in comparison to those of Newtonian flow (Gyr \& Bewersdorff, 1995; Virk, 1975). In the case in which no DR occurs, the velocity profiles can be devided into three layers: the viscous sublayer $\left(0<y^{+}<5\right)$, the buffer layer $\left(5<y^{+}<30\right)$, and the logarithmic layer or the turbulent core $\left(y^{+}>30\right)$. Virk (1971) detected an elastic layer between the viscous sublayer and the buffer layer if DR occurred; subsequently, many experimental studies described in the literature confirmed its appearance. The velocity profile in the elastic layer follows Virk's asymptote (Virk, 1971)

$$
\bar{u}^{+}=11.7 \ln y^{*}-17.0 \text {. }
$$

In the turbulent core of a non-maximum drag reducing flow, the velocity follows a log law with the Newtonian slope, but with some velocity increment $B$ compared with the Newtonian case, yielding the following equation:

$$
\bar{u}^{+}=A \ln y^{*}+5.5+B .
$$

Often $A$ is presumed to be 2.5, corresponding to the inverse of the well-known Kármán constant $(\kappa=0.4)$. The upward shift $B$ of the log-law profile has been shown to be equivalent to DR\% (Gyr \& Bewersdorff, 1995; Lumley, 1969). According to Dimant \& Poreh (1976), the relationship between the layer thickness and $B$ in Equation (22) is given by

$$
B \propto \ln \left(\frac{y_{\mathrm{e}}}{y_{\mathrm{v}}}\right),
$$

where $y_{\mathrm{v}}$ and $y_{\mathrm{e}}$ are the upper bounds of the viscous and elastic sublayers, respectively. The elastic layer successively grows with increasing DR and, at the same time, the extension of the turbulent core decreases. When DR reaches its maximum, no turbulent core appears. Therefore, we expect that the thickening of the elastic layer is responsible for the enhancement of $D R \%$. To investigate the variation in the elastic-layer thickness, we determined coefficients $A$ and $B$ involved in Equation (22) using a diagnostic plotted with respect to the mean velocity gradient. Figure 3 shows the diagnostic plotting in the form of the so-called (logarithmic) indicator function:

$$
\zeta \equiv y^{*} \frac{\mathrm{d} u^{+}}{\mathrm{d} y^{*}}=\left\{\begin{array}{cl}
y^{*} & \text { (viscous sublayer) } \\
11.7 & \text { (elastic layer) } \\
A \text { or } 1 / \kappa & \text { (logarithmic layer) }
\end{array}\right.
$$

As can be seen in the left figure of Fig. 3, the viscous sublayer, in which the indicator function has the unity gradient, ranges up to $y_{\mathrm{v}}^{*}=5$ for case 1 . For viscoelastic cases, the viscous sublayer is apparently thickened and its upper bound located at $y_{\mathrm{v}}^{*}>10$. We now employ $y_{\mathrm{v}}^{*}=11.7$, at which the linear velocity profile $\left(\bar{u}^{+}=y^{*}\right)$ and Equation (21) intersect, as proposed by Virk (1975). In the elastic layer above the viscous sublayer, obtained values of $\zeta$ for cases $3 \mathrm{~b}$ and 4 are found to exceed 11.7, which is derived from Equation (21) of Virk's asymptote for polymer solution. Note that it is not surprising that surfactant solutions can 

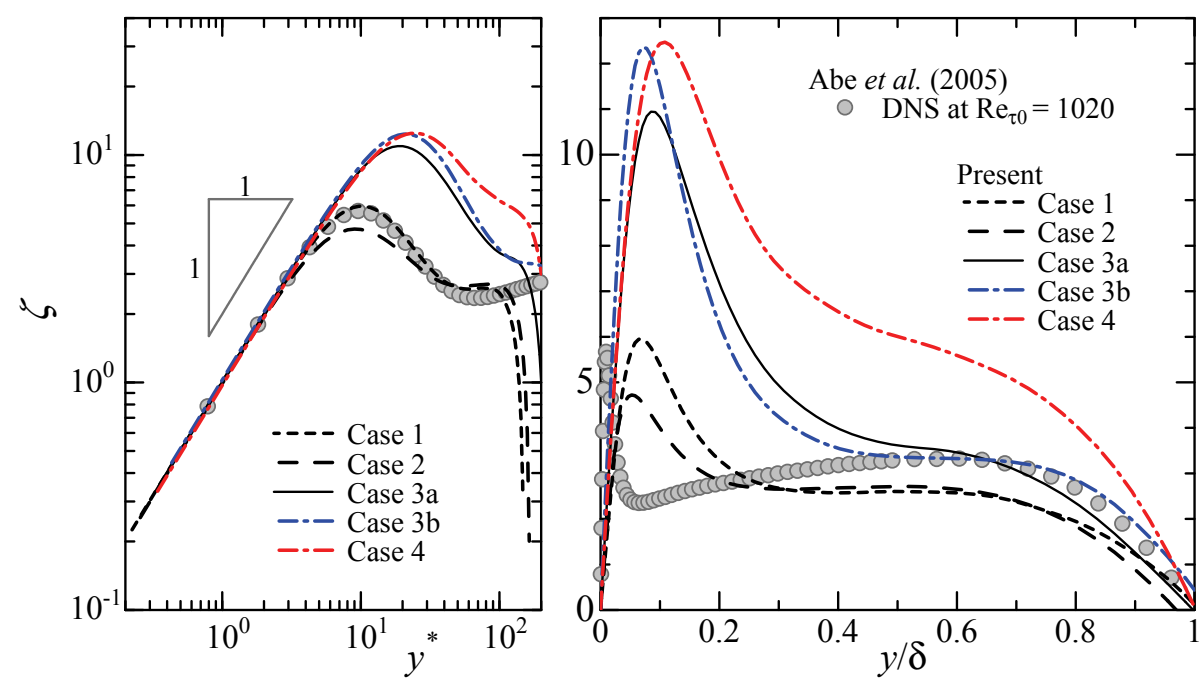

Fig. 3. Diagnostic plot, based on Equation (24), for the mean velocity profile. A profile calculated from DNS (Abe et al., 2005) for the Newtonian flow at $R e_{\tau}=1020$ is shown for comparison.

\begin{tabular}{ccccccc}
\hline Cases & $A$ & $B$ & $y_{\mathrm{v}}^{*}-y_{\mathrm{e}}^{*}$ & $y_{\mathrm{v}}^{+}-y_{\mathrm{e}}^{+}$ & $y_{\mathrm{v}} / \delta-y_{\mathrm{e}} / \delta$ & $\ln \left(y_{\mathrm{e}} / y_{\mathrm{v}}\right)$ \\
\hline 2 & 2.7 & 1.0 & $11-14$ & $9.6-12$ & $0.064-0.081$ & 0.18 \\
$3 \mathrm{a}$ & 3.6 & 6.2 & $11-35$ & $7.7-24$ & $0.051-0.16$ & 1.1 \\
$3 \mathrm{~b}$ & 3.4 & 9.7 & $11-48$ & $5.7-25$ & $0.038-0.17$ & 1.4 \\
\hline
\end{tabular}

Table 2. Coefficients $A$ and $B$ of Equation (22), and upper bounds of the viscous sublayer and the elastic layer $\left(y_{\mathrm{v}}\right.$ and $y_{\mathrm{e}}$, respectively)

lead to greater DR than that predicted by the maximum DR for polymer solutions. A number of researchers using surfactant drag-reducing additives actually reported somewhat higher levels of DR than Virk's asymptote (Zakin, 1996, and references therein).

The log-law profiles for both case $3 \mathrm{a}$ and case $3 \mathrm{~b}$ are seemingly parallel to that of case 1 , as shown in Fig. 2. It should be noted that one issue regarding the logarithmic layer remains even in the case of Newtonian flow, namely, the extent of the wall distance in which the log law is believed to be valid (Örlü et al., 2010). Also plotted in Fig. 3 is $\zeta$ calculated for the Newtonian flow at $R e_{\tau}=1020$ on the basis of the DNS database by Abe et al. (2005). A plateau of their $\zeta$ distribution can be found in a very narrow region of $y^{*}>30$ and $y / \delta\left(=y^{\star}\right)<0.2$. This is consistent with classical theory, which gives $y^{*}=30-70$ as the lower limit and $y / \delta=0.1-0.2$ as the upper limit. Some plateau regions observed in Fig. 3 are remarkably higher than $y / \delta=$ 0.2. Moreover, the $\zeta$ values also reveal that a log-law is strictly speaking absent, since $\zeta$ varies continuously as $y$ changes. Hence, the present log-law regions might not correspond to a proper logarithmic layer, since the Reynolds number in this work is too low to find a proper logarithmic layer.

Although conflicting to some extent in the above, we chose an average value in a plateau region observed in $y / \delta=0.4-0.6$, in order to give $A$ specifically for estimating $y_{\mathrm{e}}$ and $B$ of 


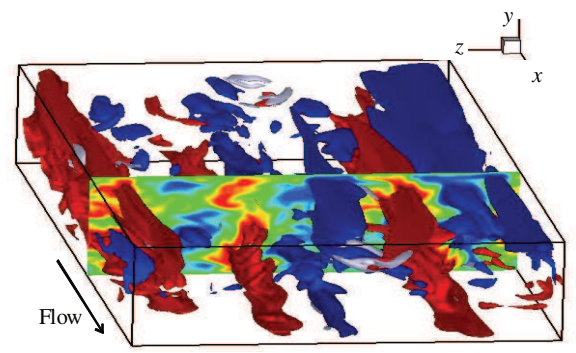

(a) Case $3 \mathrm{~b}\left(W e_{\tau}=30, \beta=0.3\right)$

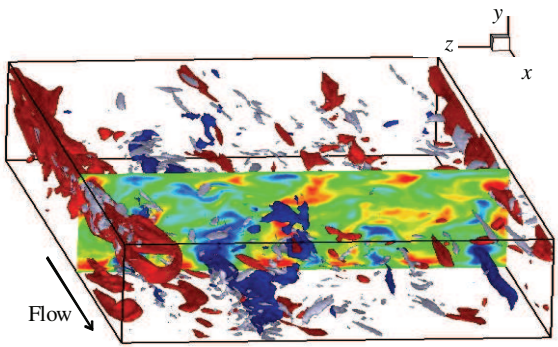

(b) Case $4\left(W e_{\tau}=40, \beta=0.5\right)$

Fig. 4. Visualization of instantaneous flow field for the highly drag-reducing flows. Iso-surfaces of streamwise velocity fluctuation are shown: red, positive; blue, negative; and white iso-surface indicates vortex.

each viscoelastic flow. In Table 2, the calculated values $A$ and $B$ for three tested cases are summarized. Also given are the extent of the elastic layer and the ratio of $y_{\mathrm{e}} / y_{\mathrm{v}}$. Here, we define $y_{e}$ as the height at which the two profiles, Equation (22) and Equation (21), intercept. In case 4 , no apparent log-law (i.e., constant $\zeta$ ) region was found between the elastic layer and the core region. Similar behavior was seen in experimental results (Warholic et al., 1999; Yu et al., 2004), where turbulent motions were suppressed and a nearly maximal level of DR was achieved. Hence, the $y_{\mathrm{e}}$ in case 4 could not be given explicitly from the above procedure. The mean velocity for case $3 b$ reveals the logarithmic profile from almost the same height as that of case 3a, as given in Fig. 2. If normalized by the outer length scale, the upper bound of the elastic layer for both cases is approximately $y_{\mathrm{e}} / \delta=0.16$. A significant difference between cases $3 a$ and $3 b$ is in the magnitude of the upward shift: $B$ in case $3 b$ is larger than that in case $3 \mathrm{a}$, and thus the obtained $D R \%$ is larger in case $3 \mathrm{~b}$. The appearance of the logarithmic layer implies a wide scale range of turbulent vortices in the flow. Therefore, the turbulent contribution to the momentum and heat transfers is not so different between these two cases at the same Weissenberg number. If we focus on the lower bound of the elastic layer, $y_{\mathrm{v}} / \delta$ of case $3 \mathrm{~b}$ is found to be shifted toward the wall compared with that of case $3 \mathrm{a}$ : namely, the viscous sublayer becomes thinner as $\beta$ decreases. We deduce that the high $D R \%$, which has been obtained in case $3 b$, is attributable to a remarkable decrease of the effective viscosity rather than the suppression of turbulent motions, thus inducing a thinning of the viscous sublayer by a relative decrease of $\beta$ (cf. Equation (23)). Two aspects of the coupling between fluid rheology and amount of DR have already been identified and discussed in detail in Tsukahara et al. (2011a): the first aspect is the reduced contribution of turbulence in a high- $W e_{\tau}$ flow, and the second is the decreased effective viscosity in a low- $\beta$ fluid. Through these two aspects, the DR should be enhanced.

The instantaneous contour surface of the streamwise velocity fluctuation is visualized for cases $3 \mathrm{~b}$ and 4 in Figs. 4(a) and (b), respectively. Also shown are negative regions of the second invariant of the deformation tensor $\left(I I^{\prime}=\partial u_{i}^{\prime} / \partial x_{j} \times u_{j}^{\prime} / \partial x_{i}\right)$, representing vortical motions. As discussed above, the vortical motions remain to occur for case $3 b$, while they are well damped for case 4 . In the latter case, the small-scale eddies were suppressed, and the streaks was remarkably enlarged. Suppression of these eddies reduces the Reynolds stress, leading to a drop in the $D R \%$. 


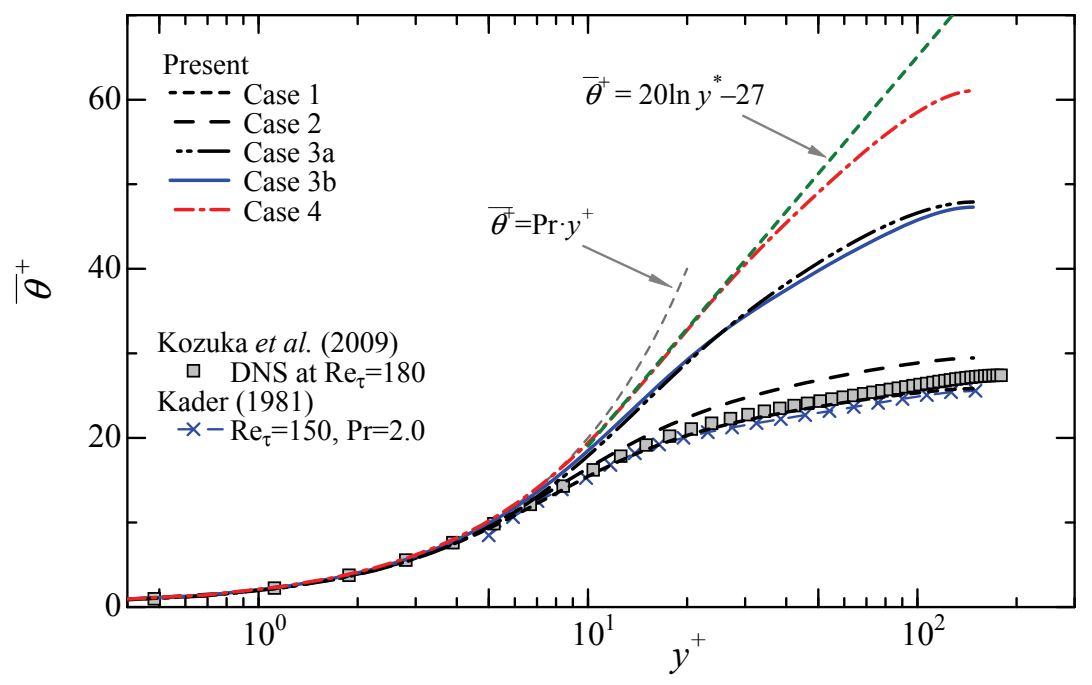

Fig. 5. Mean temperature profile in wall units for Prandtl number of 2.0. Also shown are a profile for Newtonian flow at the same Prandtl number obtained by Kozuka et al. (2009), but different $R e_{\tau}$; and one estimated by the empirical function of Kader (1981). The green broken line is a fitting curve for case 4 .

In the following sections, we analyze whether the temperature field, as well as the reduction in heat-transfer rate, undergoes different modulation depending on these aspects.

\subsection{Mean temperature profile}

The dimensionless mean temperature distribution is given in Fig. 5 for $\operatorname{Pr}=2.0$, compared with the empirical formulas of Kader (1981) for the Newtonian turbulent flow. Here, note that the abscissa is $y^{+}$in stead of $y^{*}$. The present result in case 1 (the Newtonian flow) is in good agreement with the empirical formulas and also with the DNS data of Kozuka et al. (2009), who performed simulations with fine grids (e.g., $\Delta x^{+} \approx 1$ ) at high $\operatorname{Pr}$ up to 10 . One interesting aspect of the temperature profiles shown in Fig. 5 is that the whole cross section is affected by the DR effects: namely, there is no noticeable log-law region in the turbulent core $\left(y_{\mathrm{e}}<y<\delta\right)$. The thermal buffer layer (analogous to the elastic layer) for case 4 seems to be reasonably well represented by a straight line in the semi-logarithmic coordinate, with a slope of approximately 20 in this case, that is,

$$
\bar{\theta}^{+}=20 \ln y^{+}-27 \text {. }
$$

We can compare our results with the asymptotic formula obtained by Gasljevic et al. (2007). They measured temperature profiles for various kinds of polymer and surfactant solutions. Although the Prandtl numbers in their works ranged from 6 to 9 (values for water), their results, at least qualitatively, are comparable to ours. Their obtained slope of the logarithmic temperature profile in the elastic layer was 69 for a relatively high concentration of polymer solution with $\mathrm{Pr}=6.6$. On the basis of this value, we can estimate a predicted slope of 21 for $\operatorname{Pr}=2$, which is comparable to our result (see Fig. 5). As for a surfactant solution, Gasljevic et al. (2007) showed a significantly sharp slope up to 210, corresponding to 65 for 


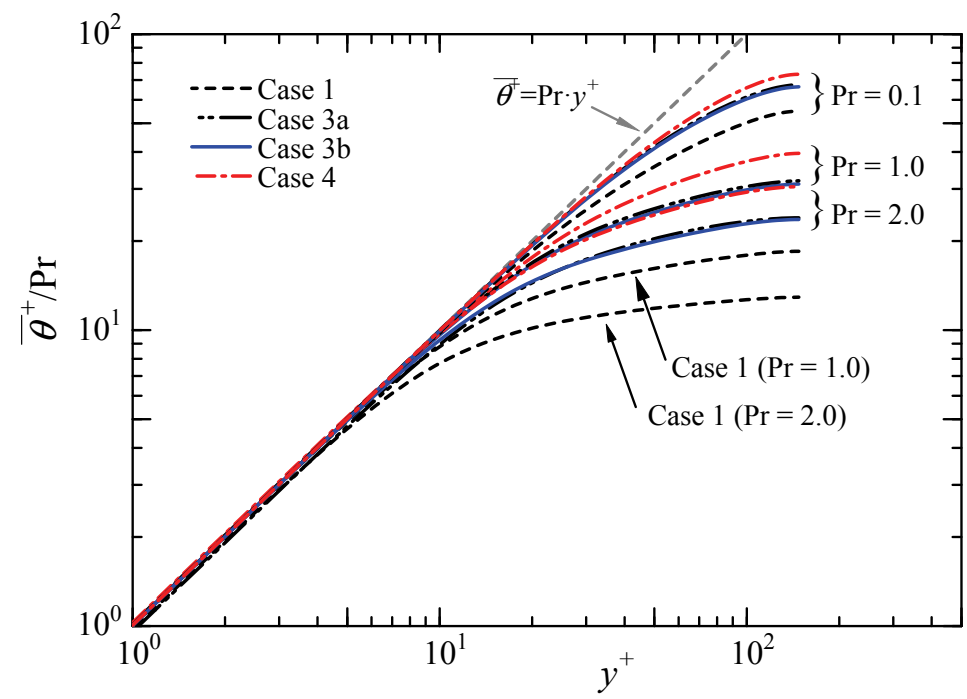

Fig. 6. Mean temperature profile in wall units for various Prandtl numbers with an emphasis on the conductive-sublayer region.

$\operatorname{Pr}=2$. They concluded that this increased slope was attributable to an apparent thickening of the viscous sublayer. However, the viscous-sublayer thickening is still somewhat elusive, as they also commented, because of difficulty in the near-wall measurement. It is challenging to explain this large difference between their temperature profiles obtained with the surfactant solution and ours. Regarding this issue, one can only conclude from the present study that a more extensive DNS database is needed over a wide range of flow conditions and Prandtl numbers.

The mean temperature profile is plotted again in Fig. 6 with an emphasis on the conductive sublayer, including results at $\mathrm{Pr}=0.1$ and 1.0. It is well known that the near-wall temperature variation can be expanded in terms of $y^{+}$as follows:

$$
\bar{\theta}^{+}=\operatorname{Pr} \cdot y^{+}+\cdots
$$

which is clearly shown in Fig. 6. It can be seen from the figure that the conductive sublayer penetrates more deeply into the core region with a decrease of the Prandtl number. This figure further indicates that thickening of the conductive sublayer occurs due to DR. At $\operatorname{Pr}=1.0$, the conductive sublayer is evident up to $y^{+} \approx 15$ for the viscoelastic flows but $y^{+} \approx 5$ for case 1 , suggesting that, in the elastic layer, the heat conduction should be dominant rather than the turbulent heat transfer. Significant upward shifts of the profile for the viscoelastic flows can be observed in the outer region, while for $\operatorname{Pr}=0.1$ the wider conductive sublayer (up to $y^{+} \approx 20$ ) appears independently of the cases. Above the conductive layer, $y^{+}>5$ for $\mathrm{Pr}=1-2\left(y^{+}>30\right.$ for $\operatorname{Pr}=0.1$, Weissenberg-number dependence was clearly observed. It is interesting to note that $\bar{\theta}^{+}$of case $3 \mathrm{~b}$ is comparable to, or slightly lower than, that of case $3 \mathrm{a}$ in the whole region of the channel (see Figs. 5 and 6). As mentioned earlier, the turbulent contribution to the heat transfer is at the same level in both cases because of the same Weissenberg number. Again, note that, in case $3 b$, the viscous sublayer is relatively wide because of a small $\eta_{\text {eff }}$, which gives 


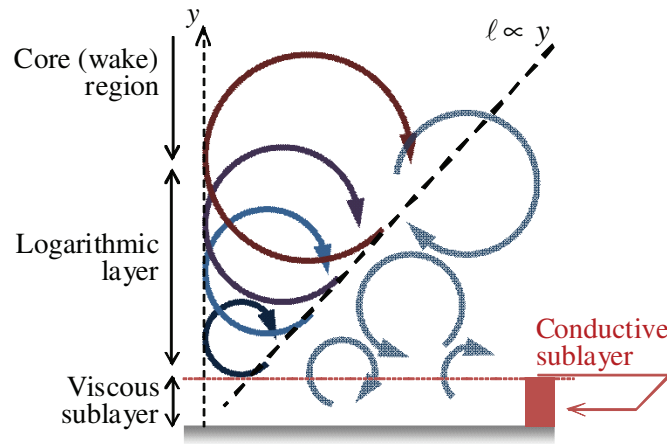

(a) Case 1: Newtonian flow

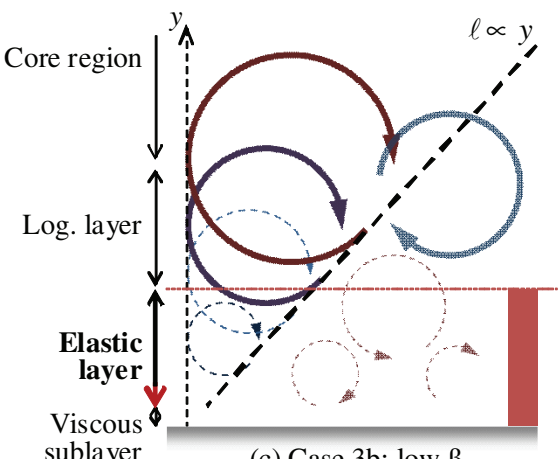

(c) Case 3b: low $B$

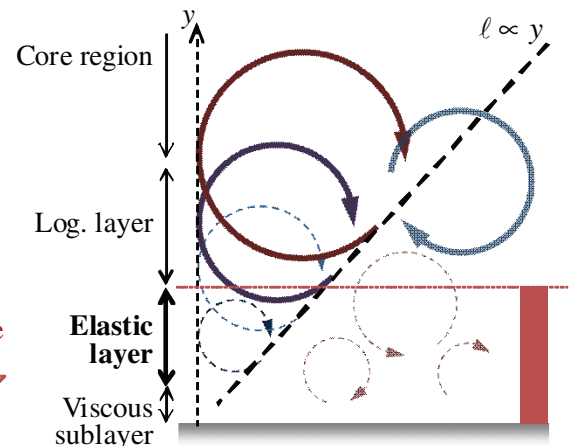

(b) Case 3a: viscoelastic flow

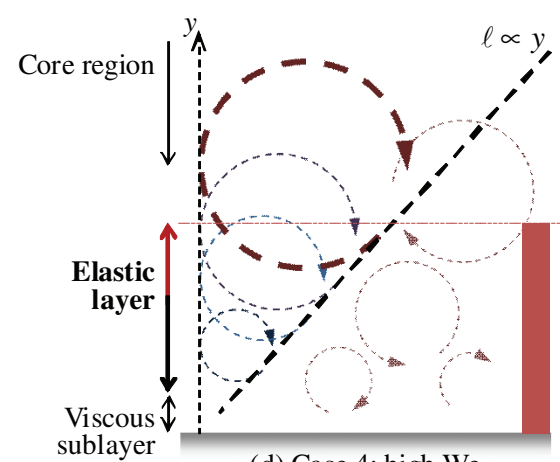

(d) Case 4: high $\mathrm{We}_{\mathrm{t}}$

Fig. 7. Schematic view of relationship between several layers and multiscale turbulent eddies affected by drag-reducing additives. A thick dashed line denotes typical eddy scales proportional to the distance from the wall, on the basis of the mixing-length theory. Red bar denotes the conductive sublayer for each relevant flow in the case of the unity Prandtl number.

rise to enhancement of DR by as much as $69 \%$. For these reasons, the obtained values of $\bar{\theta}^{+}$ as well as the heat-transfer reduction rate (mentioned later) showed less discrepancy between cases $3 \mathrm{a}$ and $3 \mathrm{~b}$. A similar conclusion is valid for other Prandtl numbers.

The analysis presented earlier has revealed important features of flow with drag reduction that can be enhanced by two factors: the suppressions of turbulence by increasing $W e_{\tau}$ and of effective viscosity by decreasing $\beta$. It has shown that it is necessary to distinguish between these effects for heat transfer of drag-reducing flow. Figure 7 schematically illustrates modulated wall turbulence for each fluid case. In the Newtonian turbulent flow as given in Fig. 7(a), we know that there is no relevant length other than the wall-normal height $y$ in the logarithmic layer (according to mixing length theory), and hence the energy-containing large-scale eddies should scale roughly with $y$. If DR occurs by the use of additives, small eddies near the wall can be damped because of the elasticity with a longer relaxation time relative to the turblence time scale, and then the elastic layer appears there (see Fig. $7(\mathrm{~b})$ ). The extent of the elastic layer will expand toward the wall with a lower $\beta$, while it will be dominant far away from the wall, instead of the logarithmic layer, in the case of high $W e_{\tau}$. In 


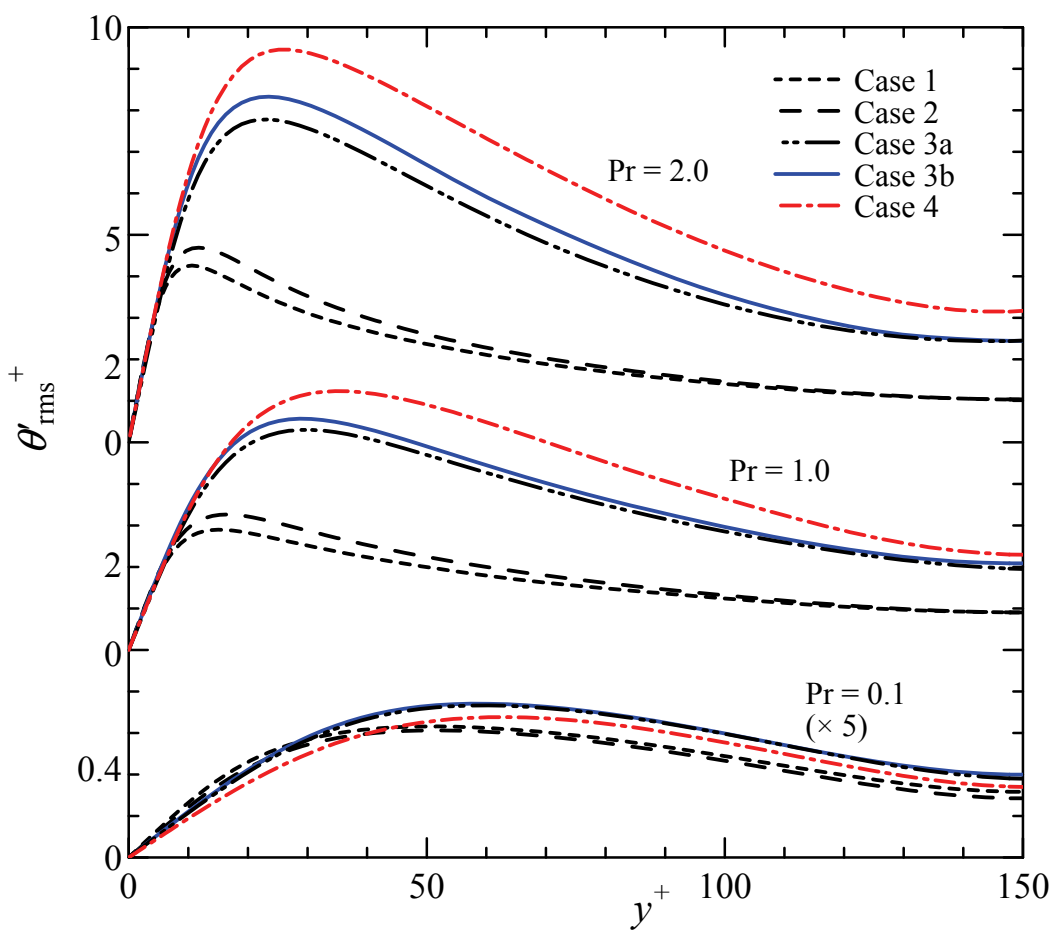

Fig. 8. Temperature variance in wall units for various Prandtl numbers.

Fig. 7, the expected conductive sublayer for each case is depicted, showing the same height for cases $3 a$ and $3 b$.

\subsection{Temperature variance}

The root-mean-square value of temperature fluctuation, namely, the temperature variance $\theta^{\prime}{ }_{\text {rms }}$, normalized by the friction temperature $T_{\tau}$ is shown in Fig. 8. We can clearly observe that $\theta_{\text {rms }}^{\prime+}$ for $P r=1.0$ and 2.0 increases compared with the Newtonian flow (case 1) as $D R \%$ increases. The values in case $3 \mathrm{~b}$ are larger than those obtained in case $3 \mathrm{a}$ and this difference is more prominent for $\mathrm{Pr}=2.0$, but it is worth noting that the peak position for cases $3 \mathrm{a}$ and $3 \mathrm{~b}$ does not exhibit significant variation. The peak of $\theta_{\mathrm{rms}}^{\prime+}$ for drag-reducing flow shifts away from the wall with variation of $W e_{\tau}$. For $P r=1.0$, the peak is located at $y^{+} \approx 29$ for both cases $3 \mathrm{a}$ and $3 \mathrm{~b}\left(W e_{\tau}=30\right)$, and at $y^{+} \approx 35$ for case $4\left(W e_{\tau}=40\right)$, so that it does not correspond to the maximum location of the streamwise turbulent intensity, $y^{+} \approx 18-25$ (figure not shown here; cf. Tsukahara et al., 2011a). As for a higher Prandtl number of $\operatorname{Pr}=2.0$, the peaks are located at $y^{+}=22-25$, which are very close, but do not coincide exactly with the locations of maximum $u_{\mathrm{rms}}^{\prime}$. In contrast, when $\mathrm{Pr}=0.1$, the conductive sublayer dominates, the peak of $\theta_{\text {rms }}^{\prime+}$ being located at $y^{+}=40-60$. The behavior and magnitude of $\theta_{\text {rms }}^{\prime+}$ for $\operatorname{Pr}=0.1$ show a collapse to the Newtonian case in the whole region irrespective of the fluid parameters, especially $\beta$. This indicates that the heat transport with a low Prandtl number is less dependent on the DR turbulent flow, which is similar to the Newtonian case. For this 


\begin{tabular}{|c|c|c|c|c|}
\hline \multirow[b]{2}{*}{$\mathrm{Pr}$} & \multicolumn{3}{|c|}{$H T R \%$} & $H T R \% / D R \%$ \\
\hline & 0.1 & 1.0 & 2.0 & $\begin{array}{lll}0.1 & 1.0 & 2.0\end{array}$ \\
\hline Case 2 & $8.0 \%$ & $16.6 \%$ & $16.3 \%$ & $\begin{array}{lll}0.39 & 0.80 & 0.79\end{array}$ \\
\hline Case $3 a$ & $49.9 \%$ & $58.5 \%$ & $62.3 \%$ & $\begin{array}{lll}0.79 & 0.93 & 0.99\end{array}$ \\
\hline Case $3 b$ & $47.2 \%$ & $57.3 \%$ & $64.9 \%$ & $\begin{array}{lll}0.68 & 0.82 & 0.93\end{array}$ \\
\hline Case 4 & $54.0 \%$ & $69.3 \%$ & $72.8 \%$ & 0.750 .971 .02 \\
\hline
\end{tabular}

Table 3. Heat-transfer reduction rates and ratio relative to drag-reduction rate

reason, the magnitude of $H T R \%$ obtained at $P r=0.1$ was relatively low compared with $D R \%$, as shown below.

Figure 8 further indicates that $\theta_{\mathrm{rms}}^{\prime+}$ in case 2 is slightly increased from that in case $1\left(5<y^{+}<\right.$ 70). It can be considered that the influence of the turbulence modulation due to the fluid viscoelasticity occurs there and does not exist in the core region $\left(70<y^{+}\right)$.

\subsection{Reduction rate of heat transfer}

Table 3 shows the percentage of heat-transfer reduction, $H T R \%$, and the ratio of HTR to DR. The rate of $H T R \%$ is calculated with the following equation:

$$
H T R \%=\frac{N u_{\mathrm{K}}-N u}{N u_{\mathrm{K}}} \times 100 \%
$$

where $N u_{\mathrm{K}}$ is the Nusselt number at the same bulk Reynolds number predicted by an empirical correlation function for Newtonian fluid:

$$
N u_{\mathrm{K}}=0.020 \operatorname{Pr}^{0.5} \operatorname{Re}_{\mathrm{m}}^{0.8} .
$$

This equation has often been used for evaluating heat-transfer correlations in channel flow. Note that we applied the coefficient 0.020 , which was recommended by Tsukahara et al. (2006), in place of 0.022 originally given by Kays \& Crawford (1980); however, we used 0.025 for $\mathrm{Pr}=0.1$ to ensure a consistency with the Newtonian case.

For a unit value of Prandtl number $(P r=1.0)$, the obtained $H T R \%$ is at the same order of magnitude as $D R \%$ in each case (see Table 3). As described previously, there are two types of factor causing DR. One is the suppression of turbulence under high $W e_{\tau}$ (e.g. case 4 in particular), and the other is the diminution in effective viscosity under low $\beta$ (case $3 b$ ). We can expect that the HTR in case 4 should also be enhanced, giving rise to a high $H T R \%$, because the turbulent motion promotes heat transfer as well as momentum transfer. In contrast, in case $3 b$, no significant change in $H T R \%$ was observed compared with that in case $3 a$, whereas the difference of $D R \%$ between the cases was relatively large. Both $D R \%$ and $H T R \%$ were increased as $W e_{\tau}$ was increased at a constant $\beta$, while only $D R \%$, rather than both, was increased with decreasing $\beta$. From the comparison with other Prandtl numbers, a similar tendency can be observed: the highest-HTR\% flow was in case 4 , and case $3 \mathrm{~b}$ showed almost identical $H T R \%$ with that in case $3 a$.

As can be seen from Table 3, the obtained values of $H T R \%$ for $P r=0.1$ are much smaller than $D R \%$ and $H T R \%$ for moderate Prandtl numbers. This is due to the low Prandtl-number effect, as discussed in section 3.6, where we examine the statistics related to turbulent heat flux. The HTR-to-DR ratio is also shown in Table 3, showing values smaller than 1 except for case 4 at a relatively high Prandtl number. According to the results, the fluid condition in case $3 \mathrm{~b}$ can be 


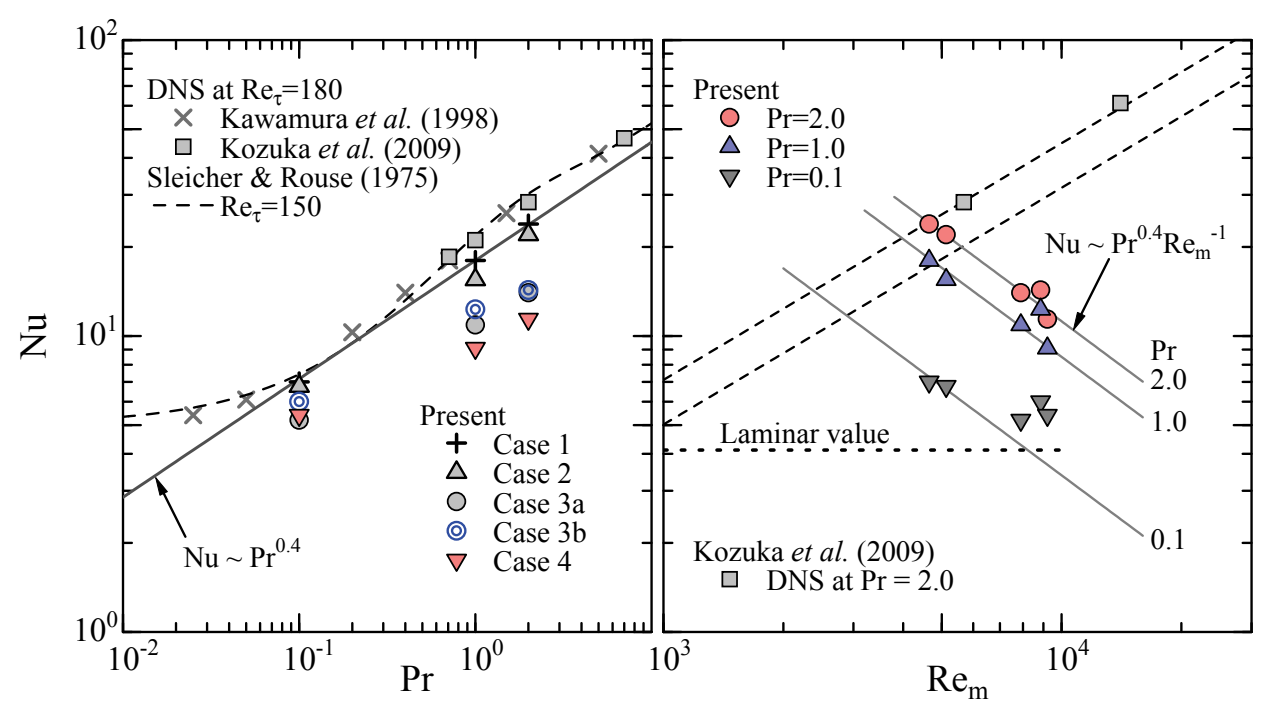

Fig. 9. Relationship between Nusselt and Prandtl numbers. DNS results by other researchers and a turbulent relationship for Newtonian flow are shown for comparison. Relation between Nusselt and Reynolds numbers. The laminar value of 4.12 and a turbulent relationship for Newtonian flow are shown for comparison.

adequate to avoid attenuation of turbulent heat transfer. However, the low Prandtl-number condition might not be practically interesting, since water (with $\operatorname{Pr}=5-10$ ) is often used as the solvent of drag-reducing flows. Aguilar et al. (1999) experimentally observed that, in drag-reducing pipe flow, the HTR-to-DR ratio decreased at higher Reynolds number and stabilized at a value of 1.14 for $R e_{\mathrm{m}}>10^{4}$. Our results showed much lower values than their measurements, but exhibited certain Prandtl-number dependence, that is, the HTR-to-DR ratio was a function of the Prandtl number.

Figure 9 shows the Prandtl-number and Reynolds-number dependences of the Nusselt number. It is practically important to compare the results for the heat transfer coefficient in drag-reducing flow with those predicted by widely used empirical correlations for Newtonian turbulent flows. The empirical correlation in terms of the $\operatorname{Pr}$ dependence suggested by Sleicher et al. (1975) is shown as a dotted line in the left figure. Note that this correlation is originally for the pipe flow; moreover, the present Reynolds number is smaller than its applicable range. The present results are lower than the correlation because of the low Reynolds-number effect. We also present a fitting curve of $\operatorname{Pr}^{0.4}$ shown by the solid line in the same figure. The results for case 1 collapse to this relationship as well as other DNS data (Kawamura et al., 1998; Kozuka et al., 2009), although a slight absolute discrepancy arises because of the difference in $R e_{\tau}$. As for the viscoelastic flows, the obtained $N u$ are smaller than the correlation, especially at moderate Prandtl numbers. It is interesting to note that the correlation of $N u \propto \operatorname{Pr}^{0.4}$ is still applicable in the range of $\operatorname{Pr}=1-2$, even for the drag-reducing flows.

We plot in the right figure ( $N u$ versus $R e_{\mathrm{m}}$ ) the corresponding values of $N u_{\mathrm{K}}$ for Newtonian turbulent flow predicted by Equation (28). The relationship in case 1 (at $R e_{\mathrm{m}}=4650$ ) shows good agreement with the empirical correlation. It is found that in viscoelastic flow 
$\mathrm{Nu}$ decreases as $R e_{\mathrm{m}}$ increases, revealing a trend quantitatively opposite to that estimated by the correlation as the following form:

$$
N u \propto \operatorname{Pr}^{0.4} \operatorname{Re}_{\mathrm{m}}^{-1} .
$$

It is clearly confirmed from Fig. 9 that Equation (29) shows much better correlation of the data at $P r=1-2$ for cases 2, 3a, and 4 (i.e., varying $W e_{\tau}$ with a constant $\beta$ ). The obtained $N u$ in case 2 (at $R e_{\mathrm{m}}=8860$ ) is significantly larger than that in the Equation (29). This also suggests that the decrease of $\beta$ gives rise to $D R \%$ with relatively small $H T R \%$ compared to a case of increasing $W e_{\tau}$. The values at $\mathrm{Pr}=0.1$ are much larger than those with Equation (29), approaching the laminar value of $N u=4.12$. Hence the turbulent heat transfer of drag-reducing flow at low Prandtl numbers may be qualitatively different from that for moderate Prandtl numbers. From a practical viewpoint, these findings are also useful. As the $N u$ appeared to be a unique function of $R e_{\mathrm{m}}$ and $\mathrm{Pr}$ even for a wide range of fluids (i.e., different relaxation times of viscoelastic fluid), one can readily predict the level of HTR on the basis of measurements of $D R \%$.

\subsection{Reduced contribution of turbulence to heat transfer}

As shown in Tables 1 and 3, non-negligible $D R \%$ and $H T R \%$ are obtained in case 2, although the attenuation of the momentum and heat transport seems to be small and limited in the near-wall region (see also Fig. 8). In addition, a large amount of HTR is achieved in the highly drag-reducing flow (cases 3-4), where near-wall turbulent motion is suppressed and the elastic layer develops. These features occur because the wall-normal turbulent heat flux as well as the Reynolds shear stress in the near-wall region should primarily contribute to the heat transfer and the frictional drag, in the context of the FIK identity (see Fukagata et al., 2002; 2005; Kagawa, 2008).

From Equation (16), the total and wall-normal turbulent heat flux can be obtained by ensemble averaging as follows:

$$
q_{\text {total }}=1-\int_{0}^{y^{\star}} \frac{\bar{u}}{u_{\mathrm{m}}} \mathrm{d} y^{\star}=\frac{1}{\operatorname{Re} \tau \operatorname{Pr}} \frac{\partial \bar{\theta}^{+}}{\partial y^{\star}}-\overline{v^{\prime+} \theta^{\prime+}} .
$$

By applying integration of $\int_{0}^{1}\left(1-y^{\star}\right) \mathrm{d} y^{\star}$, the above equation can be rearranged as follows:

$$
\frac{1}{2}-A=\frac{\Theta^{+}}{R e_{\tau} P r}+\int_{0}^{1}\left(1-y^{\star}\right)\left(-\overline{v^{\prime+} \theta^{\prime+}}\right) \mathrm{d} y^{\star},
$$

where

$$
A=\int_{0}^{1}\left(1-y^{\star}\right)\left[\int_{0}^{y^{\star}} \frac{\bar{u}}{u_{\mathrm{m}}} \mathrm{d} y^{\star}\right] \mathrm{d} y^{\star}, \quad \Theta^{+}=\int_{0}^{1} \bar{\theta}^{+} \mathrm{d} y^{\star} .
$$

Then, the relationship between the inverse of the Nusselt number (namely, the dimensionless thermal resistance of the flow) and the turbulence contribution (wall-normal turbulence heat flux) is given as follows:

$$
\begin{aligned}
R \equiv \frac{1}{N u} & =\frac{\theta_{\mathrm{m}}^{+}}{2 \operatorname{Re} \tau \operatorname{Pr}}=R_{\text {mean }}-R_{\mathrm{turb}} \\
R_{\text {mean }} & =\frac{\theta_{\mathrm{m}}}{2 \Theta}\left(\frac{1}{2}-A\right) \\
R_{\text {turb }} & =\frac{\theta_{\mathrm{m}}}{2 \Theta} \int_{0}^{1}\left(1-y^{\star}\right)\left(-\overline{v^{\prime+} \theta^{\prime+}}\right) \mathrm{d} y^{\star} .
\end{aligned}
$$




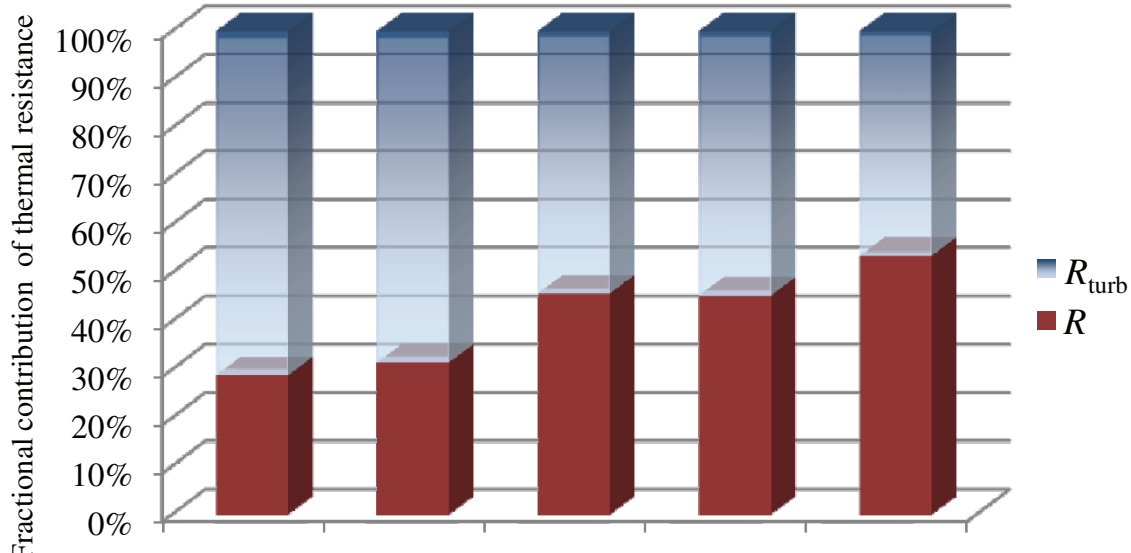

Case $1 \quad$ Case $2 \quad$ Case $3 a \quad$ Case $3 b \quad$ Case 4

Fig. 10. Fractional contribution of thermal resistance (inverse of Nusselt number) for $\operatorname{Pr}=1.0$.

Here, $R_{\text {mean }}$ corresponds to the resistance estimated from mean velocity and temperature. This identity function indicates that $R$ can be interpreted as the actual thermal resistance, which is obtained by subtraction of the negative resistance $\left(R_{\text {turb }}\right)$ due to turbulence from $R_{\text {mean }}$. For larger turbulent heat flux near the wall, the term $R_{\text {turb }}$ increases and plays an important role to decrease the thermal resistance.

In order to examine the thermal resistance under the present conditions, the components of thermal resistance in Equation (33) are shown in Fig. 10. Note that $R_{\text {mean }}$, that is, the sum of the actual thermal resistance $R$ and the turbulence contribution $R_{\text {turb }}$, is $100 \%$. Only a single Prandtl number of 1.0 is presented, but similar conclusions can be drawn for $\operatorname{Pr}=2$ 2.0. Generally, $R_{\text {turb }}$ is as much as half of $R_{\text {mean }}$ and suppresses the actual thermal resistance. An increase of $R$ should give rise to an increase of $H T R \%$. As expected, the viscoelastic flows reveal smaller fractions of $R_{\text {turb }}$ relative to the Newtonian flow of case 1 , It is interesting to note that no difference is found in the results between cases $3 a$ and $3 b$, where the same Weissenberg number is given. This is consistent with $H T R \%$, which is almost identical for both cases. In Fig. 10, $R_{\text {turb }}$ is apparently decreased as $W e_{\tau}$ changes from 0 to $10 \rightarrow 30 \rightarrow 40$. It can be concluded that the actual thermal resistance significantly depends on the Weissenberg number. In the following section, the cross correlation with respect to velocity and temperature fluctuations is discussed to investigate the diminution of the wall-normal turbulent heat flux contained in the component shown in Equation (35).

\subsection{Cross-correlation coefficients.}

Figure 11 shows the cross-correlation coefficient between the fluctuating velocity in the streamwise direction and the fluctuating temperature. This coefficient is defined as follows:

$$
R_{u \theta}=\frac{\overline{u^{\prime} \theta^{\prime}}}{u_{\mathrm{rms}}^{\prime} \theta_{\mathrm{rms}}^{\prime}} .
$$

The profile of $R_{u \theta}$ as a function of $y^{+}$is reported in Fig. 11. The $R_{u \theta}$ for the viscoelastic flows is fairly constant from 0.8 to 0.96 in most of the channel, while that in case 1 decreases monotonically there. This result means that, even in the outer region, the 

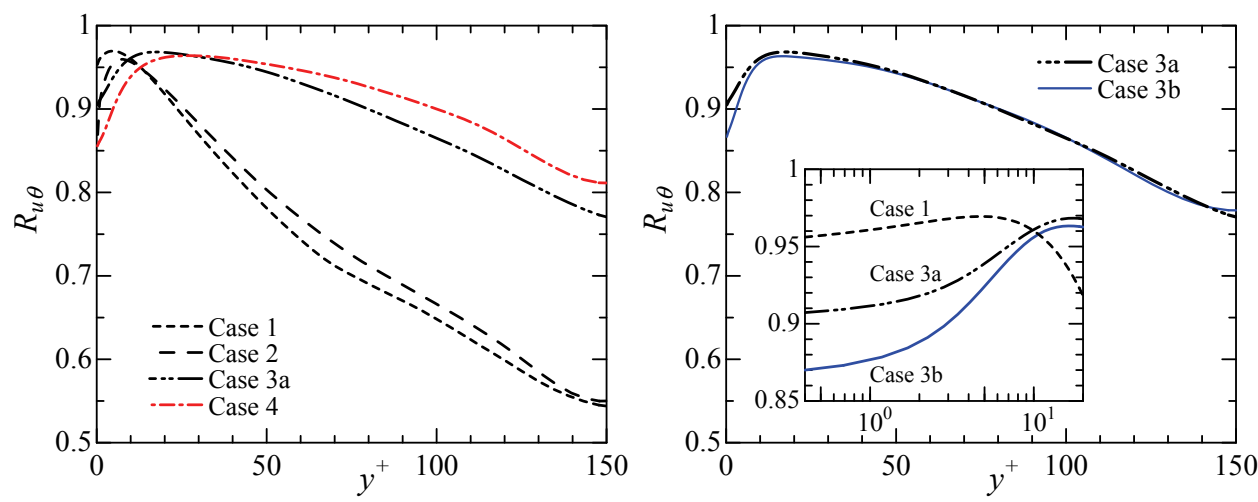

Fig. 11. Cross-correlation coefficient between fluctuations of $u^{\prime}$ and $\theta^{\prime}$ for $\operatorname{Pr}=1.0$.
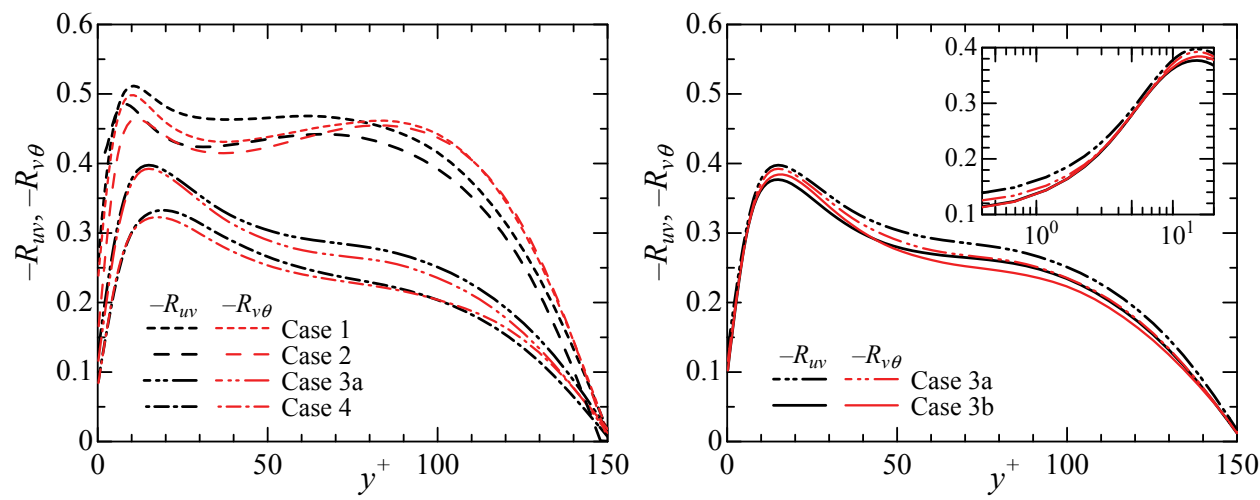

Fig. 12. Same as Fig. 11 but for $v^{\prime}$ and $u^{\prime}$, or $v^{\prime}$ and $\theta^{\prime}$.

temperature fluctuations are better correlated with the streamwise velocity fluctuations than the Newtonian case. Also note that the good match between cases $3 a$ and $3 b$ appears in the entire channel except in the vicinity of the wall, namely, in the viscous sublayer. This is consistent with above discussions in the sense that the cases are different in terms of the viscous-sublayer thickness and that the mean temperature profiles are comparable when scaled with $y^{+}$, not $y^{*}$.

As mentioned above, the wall-normal turbulent heat flux is reduced for high-Weissenberg-number flows, despite the increased temperature variance (shown in Fig. 8). It can thus be conjectured that the turbulent heat flux of $-\overline{v^{\prime} \theta^{\prime}}$ should be influenced by the loss of correlation between the two variables. Fig. 12 shows the cross-correlation coefficients of the wall-normal turbulent heat flux and of the Reynolds shear stress:

$$
R_{v \theta}=\frac{\overline{v^{\prime} \theta^{\prime}}}{v_{\mathrm{rms}}^{\prime} \theta_{\mathrm{rms}}^{\prime}}, \quad R_{u v}=\frac{\overline{u^{\prime} v^{\prime}}}{u_{\mathrm{rms}}^{\prime} v_{\mathrm{rms}}^{\prime}} .
$$

For cases 3-4, both $R_{v \theta}$ and $R_{u v}$ are much smaller than those in case 1, throughout the channel. The peak values are almost $20 \%-30 \%$ lower than the ones obtained in case 1 . The profiles of 
$R_{v \theta}$ and $R_{u v}$ for each case exhibit similar shapes throughout the channel, which also implies similarity between the variations of $-\overline{v^{\prime} \theta^{\prime}}$ and $-\overline{u^{\prime} v^{\prime}}$ affected by DR. These features at $\operatorname{Pr}=1.0$ can be seen also at the other Prandtl numbers (figure not shown) and also agree well with those of experimental results and DNS for water (Gupta et al., 2005; Li et al., 2004a). This less correlation between $\theta^{\prime}$ and $v^{\prime}$ is responsible for the decrease of the wall-normal turbulent heat flux and the increase of $H T R \%$, in the same way that the decrease of the Reynolds shear stress due to the lower correlation between $u^{\prime}$ and $v^{\prime}$ should be responsible for $D R \%$.

\section{Conclusion}

A series of direct numerical simulations (DNSs) of turbulent heat transfer in a channel flow under the uniform heat-flux condition have been performed at low friction Reynolds number $\left(R e_{\tau}=150\right)$ and various Prandtl numbers in the range of $\operatorname{Pr}=0.1$ to 2.0. In order to simulate viscoelastic fluids exhibiting drag reduction, the Giesekus constitutive equation was employed, and we considered two rheological parameters of the Weissenberg number $\left(W e_{\tau}\right)$, which characterizes the relaxation time of the fluid, and the viscosity ratio $(\beta)$ of the solvent viscosity to the total zero-shear rate solution viscosity. Several statistical turbulence quantities including the mean and fluctuating temperatures, the Nusselt number $(\mathrm{Nu})$, and the cross-correlation coefficients were obtained and analyzed with respect to their dependence on the parameters as well as the obtained drag-reduction rate $(D R \%)$ and heat-transfer reduction rate $(H T R \%)$.

The following conclusion was drawn in this study. High $D R \%$ was achieved by two factors: (i) the suppressed contribution of turbulence due to high $W e_{\tau}$ and (ii) the decrease of the effective viscosity due to low $\beta$. A difference in the rate of increase of $H T R \%$ between these factors was found. This is attributed to the different dependencies of the elastic layer on $\beta$ and $W e_{\tau}$. A case with low $\beta$ gives rise to high $D R \%$ with low $H T R \%$ compared with those obtained with high $W e_{\tau}$. Differences were also found in various statistical data such as the mean-temperature and the temperature-variance profiles. Moreover, it was found that in the drag-reducing flow $N u$ should decrease as $R e_{\mathrm{m}}$ increases, revealing the form of Equation (29) when $W e_{\tau}$ was varied with a fixed $\beta(=0.5)$. For a Prandtl number as low as 0.1 , the obtained $H T R \%$ was significantly small compared with the magnitude of $D R \%$ irrespective of difference in the rheological parameters.

Although the present Reynolds and Prandtl numbers were considerably lower than those corresponding to conditions under which DR in practical flow systems is observed with dilute additive solutions, we have elucidated the dependencies of DR and HTR on rheological parameters through parametric DNS study. More extended DNS studies for higher Reynolds and Prandtl numbers with a wide range of Weissenberg numbers might be necessary. The above conclusions have been drawn for very limited geometries such as straight duct and pipe. In terms of industrial applications, viscoelastic flows through complicated geometries should be investigated with detailed simulations. Moreover, modeling approaches for viscoelastic turbulent flows have to be developed and these are essentially of RANS (Reynolds-averaged Navier-Stokes) techniques and of LES (large-eddy simulation). DNS studies on these issues are ongoing (Kawamoto et al., 2010; Pinho et al., 2008; Tsukahara et al., 2011c) and the observations in these works will be valuable for those studying such complicated flows using RANS and LES. 


\section{Acknowledgments}

The present computations were performed with the use of supercomputing resources at Cyberscience Center of Tohoku University and Earth Simulator (ES2) at the Japan Agency for Marine-Earth Science and Technology. We also gratefully acknowledge the assistance of Mr. Takahiro Ishigami, who was a Master's course student at Tokyo University of Science. This paper is a revised and expanded version of a paper entitled "Influence of rheological parameters on turbulent heat transfer in drag-reducing viscoelastic channel flow," presented at the Fourteenth International Heat Transfer Conference (Tsukahara et al., 2010).

\section{References}

Abe, H., Kawamura, H., \& Matsuo, Y. (1998). Surface heat-flux fluctuations in a turbulent channel flow up to $R e_{\tau}=1020$ with $\operatorname{Pr}=0.025$ and 0.71 , International Journal of Heat and Fluid Flow, Vol. 25, 404-419.

Aguilar, G., Gasljevic, K., \& Matthys, E.F. (1999). Coupling between heat and momentum transfer mechanisms for drag-reducing polymer and surfactant solutions, Transactions of ASME C: Journal of Heat Transfer, Vol. 121, 796-802.

Aguilar, G., Gasljevic, K., \& Matthys, E.F. (2001). Asymptotes of maximum friction and heat transfer reductions for drag-reducing surfactant solutions, International Journal of Heat and Mass Transfer, Vol. 44, 2835-2843.

Aly, W.I, Inaba, H., Haruki, N., \& Horibe, A. (1999). Drag and heat transfer reduction phenomena of drag-reducing surfactant solutions in straight and helical pipes, Transactions of ASME C: Journal of Heat Transfer, Vol. 128, 800-810.

Basombrío, F.G., Buscaglia, G.C., \& Dari, E.A. (1991). A new approach for the FEM simulation of viscoelastic flows, Journal of Non-Newtonian Fluid Mechanics, Vol. 39, 189-206.

Bird, R.B. (1995). Constitutive equations for polymeric liquids, Annual Review of Fluid Mechanics, Vol. 27, 169-193.

Cho, Y.I. \& Hartnett, J.P. (1982). Non-Newtonian fluids in circular pipe flow. Advances in Heat Transfer, Vol. 15, 59-141.

Dean, R.D. (1978). Reynolds number dependence of skin friction and other bulk flow variables in two-dimensional rectangular duct flow, Transactions of ASME I: Journal of Fluids Engineering, Vol. 100, 215-223..

Den Toonder, J.M.J., Hulsen, M.A., Kuiken, G.D.C., \& Nieuwstadt, F.T.M. (1997). Drag reduction by polymer additives in a turbulent pipe flow: numerical and laboratory experiments, Journal of Fluid Mechanics, Vol. 337, 193-231 .

Dimant, Y. \& Poreh, M. (1976). Heat transfer in flows with drag reduction. In: Advances in Heat Transfer, Vol. 12, Irvine, T.F. \& Hartnett, J.P., (Ed.), pp. 77-113, Academic Press, Inc., ISBN 0-12-020012-0, New York.

Dimitropoulos, C.D., Sureshkumar, R., Beris, A.N., \& Handler, R.A. (2001). Budgets of Reynolds stress, kinetic energy and streamwise enstrophy in viscoelastic turbulent channel flow, Physics of Fluids, Vol. 13, 1016-1027.

Dimitropoulos, C.D., Dubief, Y., Shaqfeh, E.S.G., Moin, P., \& Lele, S.K. (2005). Direct numerical simulation of polymer-induced drag reduction in turbulent boundary layer flow, Physics of Fluids, Vol. 17, 011705, 4 pp.

Fukagata, K., Iwamoto, K., \& Kasagi, N. (2002). Contribution of Reynolds stress distribution to the skin friction in wall-bounded flows, Physics of Fluids, Vol. 14, L73-L76. 
Fukagata, K., Iwamoto, K., \& Kasagi, N. (2005). Novel turbulence control strategy for simultaneously achieving friction drag reduction and heat transfer augmentation, In: Proceedings of Fourth International Symposium on Turbulence and Shear Flow Phenomena, Humphrey, J.A.C. et al. (Ed.), pp. 307-312, Williamsburg, USA, 27-29 June 2005.

Fortin, M. \& Fortin, M. (1989). A new approach for the FEM simulation of viscoelastic flows , Journal of Non-Newtonian Fluid Mechanics, Vol. 32, 295-310.

Gasljevic, K. \& Matthys, E.F. (1999). Improved quantification of the drag reduction phenomenon through turbulence reduction parameters, Journal of Non-Newtonian Fluid Mechanics, Vol. 84, 123-130.

Gasljevic, K., Aguilar, G., \& Matthys, E.F. (2007). Measurement of temperature profiles in turbulent pipe flow of polymer and surfactant drag-reducing solutions, Physics of Fluids, Vol. 19, 083105, 18 pp.

Giesekus, H. (1982). A simple constitutive equation for polymer fluids based on the concept of deformation-dependent tensorial mobility, Journal of Non-Newtonian Fluid Mechanics, Vol. 11, 69-109.

Gyr, A. \& Bewersdorff, H.-W. (1995). Drag reduction of turbulent flows by additives, Kluwer Academic Publisher, ISBN 978-90-481-4555-3, Dordrecht.

Gupta, V.K., Sureshkumar, R., \& Khomami, B. (2005). Passive scalar transport in polymer drag-reduced turbulent channel flow, AIChE Journal, Vol. 51, 1938-1950.

Housiadas, K.D. \& Beris, A.N. (2007). Characteristic scales and drag reduction evaluation in turbulent channel flow of nonconstant viscosity viscoelastic fluids, Physics of Fluids, Vol. 16, 1581-1586.

Hoyt, J.W. (1990). Drag reduction by polymers and surfactants, In: Viscous drag reduction in boundary layers, Bushnell, D.M. \& Hefner, J.M., (Ed.), pp. 413-432, American Institute of Aeronautics and Astronautics, Inc., ISBN 978-0-930403-66-5, Washington D.C.

Joseph, D.D. (1990). Fluid dynamics of viscoelastic liquids, Springer-Verlag, ISBN 978-0387971551, New York.

Jovanović, J., Pashtrapanska, M., Frohnapfel, B., Durst, F., Koskinen, J., \& Koskinen, K. (2006). On the mechanism responsible for turbulent drag reduction by dilute addition of high polymers: theory, experiments, simulations, and predictions, Transactions of ASME I: Journal of Fluids Engineering, Vol. 128, 118-130.

Kader, B.A. (1981). Temperature and concentration profiles in fully turbulent boundary layers, International Journal of Heat and Mass Transfer, Vol. 24, 1541-1544.

Kagawa, Y., Yu, B., Kawaguchi, Y., Kawamura, H., \& Shiraishi, Y. (2008). Turbulent heat transfer of viscoelastic fluid flow accompanied by drag reduction with DNS analysis, Progress in Computational Fluid Dynamics, Vol. 8, 477-485.

Kawaguchi, Y., Wei, J.J., Yu, B., \& Feng, Z.P. (2003). Rheological characterization of drag-reducing cationic surfactant solution: shear and elongational viscosities of dilute solutions, Proceedings of ASME/JSME 2003 4th Joint Fluids Summer Engineering Conference, pp. 721-728, Honolulu, Hawaii, USA, July 6-10, 2003.

Kawamoto, H., Tsukahara, T., Kawamura, H., \& Kawaguchi, Y. (2010). Proposal of $k-\varepsilon$ model for visco-elastic fluid flow: toward prediction of drag-reducing turbulence, Proceedings of Eighth International ERCOFTAC Symposium on Engineering Turbulence Modelling and Measurements, pp. 350-355, Marseille, France, 9-11 June, 2010.

Kawamura, H., Ohsaka, K., Abe, H. \& Yamamoto, K. (1998). DNS of turbulent heat transfer in channel flow with low to mediumhigh Prandtl number fluid, International Journal of Heat and Fluid Flow, Vol. 19, 482-491. 
Kays, W.M. \& Crawford, M.E. (1980). Convective heat and mass transfer, Second edition, McGraw-Hill Book Company, Inc., ISBN 0-07-033457-9.

Keunings, R. (1990). Progress and challenges in computational rheology, Rheological Acta, Vol. 29, 556-580.

Kim, K., Adrian, R.J., Balachandar, S., \& Sureshkumar, R. (2008). Dynamics of hairpin vortices and polymer-induced turbulent drag reduction, Physical Review Letter, Vol. 100, 134504, 4pp.

Kozuka, M., Seki, Y., \& Kawamura, H. (2009). DNS of turbulent heat transfer in a channel flow with a high spatial resolution, International Journal of Heat and Fluid Flow, Vol. 30, 514-524.

Li, C.-F., Sureshkumar, R., \& Khomami, B. (2006). Influence of rheological parameters on polymer induced turbulent drag reduction, Journal of Non-Newtonian Fluid Mechcanics, Vol. 140, 23-40.

Li, F.-C., Kawaguchi, Y., \& Hishida, K. (2004a). Investigation on the characteristics of turbulence transport for momentum and heat in a drag-reducing surfactant solution flow, Physics of Fluids, Vol. 16, 3281-3295.

Li, F.-C., Wang, D.-Z., Kawaguchi, Y., \& Hishida, K. (2004b). Simultaneous measurements of velocity and temperature fluctuations in thermal boundary layer in a drag-reducing surfactant solution flow, Experiments in Fluids, Vol. 36, 131-140.

Li, F.-C.; Kawaguchi, Y., \& Hishida, K. (2005). Structural analysis of turbulent transport in a heated drag-reducing channel flow with surfactant additives, International Journal of Heat and Mass Transfer, Vol. 48, 965-973 .

Li, P.-W., Kawaguchi, Y., \& Yabe, A. (2001). Transitional heat transfer and turbulent characteristics of drag-reducing flow through a contracted channel, Journal of Enhanced Heat Transfer, Vol. 8, 23-40.

Lumley, J.L. (1969). Drag reduction by additives, Annual Review of Fluid Mechanics, Vol. 1, 367-384.

Nadolink, R.H. \& Haigh, W.W. (1995). Bibliography on skin friction reduction with polymers and other boundary-layer additives, Applied Mechanics Reviews, Vol. 48, 351-460.

Örlü, R., Fransson, J.H.M., \& Alfredsson, P.H. (2010). On near wall measurements of wall bounded flows-The necessity of an accurate determination of the wall position, Progress in Aerospace Sciences, Vol. 46, 353-387.

Procaccia, I., L'vov, V.S., \& Benzi, R. (2008). Colloquium: Theory of drag reduction by polymers in wall-bounded turbulence, Reviews of Modern Physics, Vol. 80, 225-247.

Pinho, F.T., Li, C.F., Younis, B.A., \& Sureshkumar, R. (2008). A low Reynolds number turbulence closure for viscoelastic fluids, Journal of Non-Newtonian Fluid Mechanics, Vol. 154, 89-108.

Qi, Y., Kawaguchi, Y., Lin, Z., Ewing, M., Christensen, R.N., \& Zakin, J.L. (2001). Enhanced heat transfer of drag reducing surfactant solutions with fluted tube-in-tube heat exchanger, International Journal Heat and Mass Transfer, Vol. 44, 1495-1505.

Qi, Y., Kawaguchi, Y., Christensen, R.N., \& Zakin, J.L. (2003). Enhancing heat transfer ability of drag reducing surfactant solutions with static mixers and honeycombs, International Journal Heat and Mass Transfer, Vol. 46, 5161-5173.

Roy, A., Morozov, A., van Saarloos, W., \& Larson, R.G. (2006). Mechanism of polymer drag reduction using a low-dimensional model, Physical Review Letter, Vol. 97, 234501, 4pp. 
Sleicher, C.A. \& Rouse, M.W. (1975). A convenient correlation for heat transfer to constant and variable property fluids in turbulent pipe flow, International Journal of Heat and Fluid Flow, Vol. 18, 677-683.

Shenoy, A.V. (1984). A review on drag reduction with special reference to micellar systems, Colloid \& Polymer Science, Vol. 262, 319-337.

Sureshkumar, R. \& Beris, A.N. (1995). Effect of artificial stress diffusivity on the stability of numerical calculations and the flow dynamics of time-dependent viscoelastic flows, Journal of Non-Newtonian Fluid Mechanics, Vol. 60, 53-80.

Sureshkumar, R., Beris, A.N., \& Handler, R.A. (1997). Direct numerical simulation of the turbulent channel flow of a polymer solution, Physics of Fluids, Vol. 9, 743-755.

Tamano, S., Itoh, M., Hoshizaki, K., \& Yokota, K. (2007). Direct numerical simulation of the drag-reducing turbulent boundary layer of viscoelastic fluid, Physics of Fluids, Vol. 19, 075106, $17 \mathrm{pp}$.

Toms, B.A. (1949). Some observations on the flow of linear polymer solutions through straight tubes at large Reynolds numbers, Proceedings of the First International Congress on Rheology, Vol. 2, pp. 135-141, Netherlands, 1948, North-Holland Publishing Company, Amsterdam.

Tsukahara, T., Iwamoto, K., Kawamura, H., \& Takeda, T. (2006). DNS of heat transfer in a transitional channel flow accompanied by a turbulent puff-like structure, In: Turbulence, Heat and Mass Transfer, Vol. 5, Hanjalić, K.; Nagano, Y.; \& Jakirlić, S., (Ed.), pp. 193-196, ISBN 1-56700-229-3, Dubrovnik, Croatia, 25-29 September 2006, Begell House Inc., New York, Wallingford (UK).

Tsukahara, T., Ishigami, T., Kurano, J., \& Kawaguchi, Y., (2010). Influence of rheological parameters on turbulent heat transfer in drag-reducing viscoelastic channel flow, In: Proceedings of 14th International Heat Transfer Conference, IHTC14-23138 (DVD), 10 pp., Washington DC, USA, 8-13 August 2010.

Tsukahara, T., Ishigami, T., Yu, B., \& Kawaguchi, Y. (2011a). DNS study on viscoelastic effect in drag-reduced turbulent channel flow, Journal of Turbulence, Vol. 12, No. 13, 13 pp.

Tsukahara, T. \& Kawaguchi, Y. (2011b). Comparison of heat-transfer reduction in drag-reduced turbulent channel flows with different fluid and thermal boundary conditions, Progress in Computational Fluid Dynamics, Vol. 11, Nos. 3/4, 216-226.

Tsukahara, T., Kawase, T., \& Kawaguchi, Y. (2011c). DNS of viscoelastic turbulent channel flow with rectangular orifice at low Reynolds number, International Journal of Heat and Fluid Flow, Vol. 32, 529-538.

Virk, P.S. (1971). An elastic sublayer model for drag reduction by dilute solutions of linear macromolecules, Journal of Fluid Mechanics, Vol. 45, 417-440.

Virk, P.S. (1975). Drag reduction fundamentals, AIChE Journal, Vol. 21, 625-656.

Warholic, M., Schmidt, G., \& Hanratty, T. (1999). The influence of a drag-reducing surfactant on a turbulent velocity field. Journal of Fluid Mechanics, Vol. 388, 1-20.

White, C.M. \& Mungal, M.G. (2008). Mechanics and prediction of turbulent drag reduction with polymer additives, Annual Review Fluid Mechacnics, Vol. 40, 235-256.

Wei, J., Kawaguchi, Y., Yu, B., \& Feng, Z. (2006). Rheological characteristics and turbulent friction drag and heat transfer reductions of a very dilute cationic surfactant solution, Transactions of ASME C: J. Heat Transfer, Vol. 114, 977-983.

Yu, B. \& Kawaguchi, Y. (2004). Direct numerical simulation of viscoelastic drag-reducing flow: a faithful finite difference method, Journal of Non-Newtonian Fluid Mechanics, Vol. 116, 431-466. 
Yu, B., Li, F., \& Kawaguchi, Y. (2004). Numerical and experimental investigation of turbulent characteristics in a drag-reducing flow with surfactant additives, International Journal of Heat and Fluid Flow, Vol. 25, 961-974.

Yu, B. \& Kawaguchi, Y. (2005). DNS of fully developed turbulent heat transfer of a viscoelastic drag-reducing flow, International Journal of Heat and Mass Transfer, Vol. 48, 4569-4578.

Yu, B. \& Kawaguchi, Y. (2006). Parametric study of surfactant-induced drag reduction by DNS, International Journal of Heat and Fluid Flow, Vol. 27, 887-894.

Zhu, J. \& Rodi, W. (1991). A low dispersion and bounded convection scheme, Computer Methods in Applied Mechanics and Engineering, Vol. 92, 87-96.

Zakin, J.L., Myska, J., \& Chara, Z. (1996). New limiting drag reduction and velocity profile asymptotes for nonpolymeric additives systems, AIChE Journal, Vol. 42, 3544-3546. 


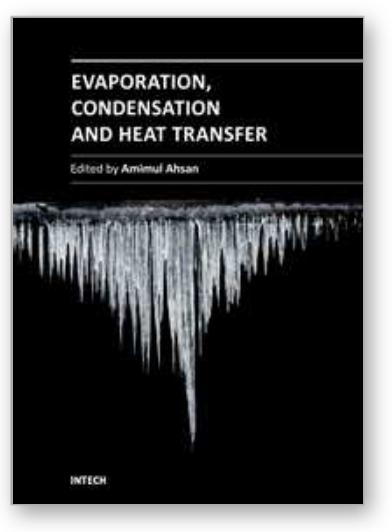

\author{
Evaporation, Condensation and Heat transfer \\ Edited by Dr. Amimul Ahsan
}

ISBN 978-953-307-583-9

Hard cover, 582 pages

Publisher InTech

Published online 12, September, 2011

Published in print edition September, 2011

The theoretical analysis and modeling of heat and mass transfer rates produced in evaporation and condensation processes are significant issues in a design of wide range of industrial processes and devices. This book includes 25 advanced and revised contributions, and it covers mainly (1) evaporation and boiling, (2) condensation and cooling, (3) heat transfer and exchanger, and (4) fluid and flow. The readers of this book will appreciate the current issues of modeling on evaporation, water vapor condensation, heat transfer and exchanger, and on fluid flow in different aspects. The approaches would be applicable in various industrial purposes as well. The advanced idea and information described here will be fruitful for the readers to find a sustainable solution in an industrialized society.

\title{
How to reference
}

In order to correctly reference this scholarly work, feel free to copy and paste the following:

Takahiro Tsukahara and Yasuo Kawaguchi (2011). Turbulent Heat Transfer in Drag-Reducing Channel Flow of Viscoelastic Fluid, Evaporation, Condensation and Heat transfer, Dr. Amimul Ahsan (Ed.), ISBN: 978-953307-583-9, InTech, Available from: http://www.intechopen.com/books/evaporation-condensation-and-heattransfer/turbulent-heat-transfer-in-drag-reducing-channel-flow-of-viscoelastic-fluid

\section{INTECH}

open science | open minds

\section{InTech Europe}

University Campus STeP Ri

Slavka Krautzeka 83/A

51000 Rijeka, Croatia

Phone: +385 (51) 770447

Fax: +385 (51) 686166

www.intechopen.com

\section{InTech China}

Unit 405, Office Block, Hotel Equatorial Shanghai

No.65, Yan An Road (West), Shanghai, 200040, China

中国上海市延安西路65号上海国际贵都大饭店办公楼405单元

Phone: +86-21-62489820

Fax: +86-21-62489821 
(C) 2011 The Author(s). Licensee IntechOpen. This chapter is distributed under the terms of the Creative Commons Attribution-NonCommercialShareAlike-3.0 License, which permits use, distribution and reproduction for non-commercial purposes, provided the original is properly cited and derivative works building on this content are distributed under the same license. 